Copyright (1996, American Institute of Aeronautics and Astronautics, Inc.

\title{
Unsteady shock-wave reflection and interaction in viscous flow with thermal and chemical nonequilibrium
}

\author{
Gregory H. Furumoto \\ California, Univ., Los Angeles \\ Xiaolin Zhong \\ California, Univ., Los Angeles \\ John C. Skiba \\ California, Univ., Los Angeles
}

\section{AIAA, Aerospace Sciences Meeting and Exhibit, 34th, Reno, NV, Jan. 15-18, 1996}

Steady shock/boundary layer interactions and self-sustained unsteady type IV shock-shock interference heating problems with nonequilibrium real gas effects were studied by numerical simulations. We emphasized the effects of internal thermochemical excitation on surface heating rates, skin friction, and flow-field unsteadiness of the viscous shock interactions. The multicomponent Navier-Stokes equations with nonequilibrium rotational, vibrational, and chemical models for five-species air were solved by a finite-volume second-order TVD scheme together with a new third-order semi-implicit Runge-Kutta scheme. For the steady shock/boundary layer interaction on a flat plate, it was found that the real gas effects reduce the size of the shock-induced separation bubble and the magnitude of the surface heating rates. Type-IV shock-shock interference heating flows with real gas effects are inherently unsteady to a degree related to the location of the jet impingement on the cylinder relative to the stagnation point. For certain impingement positions, vortices are generated and shed off near the jet impingement point. (Author) 


\title{
UNSTEADY SHOCK-WAVE REFLECTION AND INTERACTION IN VISCOUS FLOW WITH THERMAL AND CHEMICAL NONEQUILIBRIUM
}

\author{
Gregory H. Furumoto*, Xiaolin Zhong ${ }^{\dagger}$, and John C. Skiba ${ }^{\dagger}$ \\ University of California, Los Angeles, CA 90095
}

\begin{abstract}
Steady shock/boundary layer interactions and self-sustained unsteady type IV shock-shock interference heating problems with nonequilibrium real gas effects were studied by numerical simulations. The emphasis of the investigation was on the effects of internal thermo-chemical excitation on surface heating rates, skin friction, and flow field unsteadiness of the viscous shock interactions. The multicomponent Navier-Stokes equations with nonequilibrium rotational, vibrational, and chemical models for fivespecies air were solved by a finite-volume secondorder TVD scheme together with a new third-order semi-implicit Runge-Kutta scheme. For the steady shock/boundary layer interaction on a flat plate, it was found that the real gas effects reduce the size of the shock induced separation bubble and the magnitude of the surface heating rates. For the unsteady shock-shock interference heating of a pure $\mathrm{N}_{2}$ flow over a cylinder, the results showed that type-IV shock-shock interference heating flows with real gas effects are inherently unsteady. The degree of the unsteadiness is related to the location of the jet impingement on the cylinder relative to the stagnation point. For certain impingement positions, vortices are generated and shed off near the jet impingement point. This periodic shedding of the vortices contributes to the self-substained oscillations of both the jet and other parts of the flow fields. In addition, the real gas effects reduce the level of peak surface heating and peak surface pressure in the case of endothermic internal-mode excitations.
\end{abstract}

\footnotetext{
* Graduate Student Researcher, Department of Mechanical and Aerospace Engineering, Student Member AİAA

tAssistant Professor, Department of Mechanical and Aerospace Engineering, Member AIAA

† Graduate Student, Department of Mechanical and Aerospace Engineering.

Copyright (C)1996 by the American Institute of Aeronautics and Astronautics, Inc. All rights reserved.
}

\section{Introduction}

Understanding unsteady shock/boundary layer and shock-shock interference heating phenomena is vital for the design of hypersonic vehicles. Such interactions occur in many external and internal flow fields around steady and maneuvering hypersonic vehicles, such as the one shown in Figure 1. The interactions strongly affect the magnitudes of surface heating rates and skin friction. Two types of shock interactions, shock/boundary layer interactions and type IV shock interference heating, were examined in this study.

The first class of shock interactions is the shock/boundary layer interaction, where an incident oblique shock is reflected by a surface. Interaction between the incident and reflected shock waves and the boundary layer occurs. Figure 2 shows a schematic of a steady shock-wave reflection interaction with the viscous boundary layer. The large adverse pressure gradient due to the incident shock hitting the wall causes the boundary layer to separate. When the boundary layer reattaches, a reattachment compression shock is formed.

The second class of hypersonic shock interactions is the type IV shock interference interaction. Figure 3 shows a type IV interaction which is caused by an impinging oblique shock intersecting the free stream bow shock ahead of a body. This interaction creates a transmitted shock which then impinges upon the lower bow shock (due to the flow behind the initial impinging oblique shock). Behind this transmitted shock, which is weaker than either bow shock, a supersonic jet is formed in the surrounding subsonic flow. This jet impinges on the body, ending in a terminating strong shock. At the jet impingement point, extremely high surface pressures and heating rates are encountered ${ }^{[9,10,11,12]}$. As the jet flow is expanded over the surface, it once again becomes supersonic. This creates a shear layer along the body between the flow from the jet and the subsonic bulk 
flow behind the bow shock. This type of interaction has been shown to be inherently unstable ${ }^{[13,14]}$ in ideal gas flows.

To date most studies of the shock interactions have been limited to ideal gas flows for both shock/boundary layer interaction ${ }^{[20,1]}$, and type IV shock interference heating. The ideal gas type IV problem has been extensively studied experimentally and analytically $[10,17]$, and numerically $[12,13,14,11]$. However, due to the high temperatures found in these interactions, real gas effects need to be considered for many of these flow studies. Real gas effects have a noticeable impact on flow structure, such as the reduction of the shock stand off distance in a blunt body flow ${ }^{[40]}$, and parameters such as surface heating rates, which can be either reduced or enhanced depending on the nature of the chemical nonequilibrium.

Because of their importance, real gas effects have recently been the focus of several studies. For steady shock/boundary layer interactions, Ballaro and Anderson ${ }^{[4]}$, and Grumet et al. ${ }^{[5]}$ have performed numerical studies of flow at a scramjet inlet with real gas effects. In their cases, they assumed a partially dissociated freestream to simulate conditions at an inlet behind the bow shock of a vehicle. They found that the recombination of species at the wall enhanced the surface heat flux. Type IV interactions were studied numerically by Prabhu, et al. ${ }^{[16]}$ for equilibrium chemistry. More recently, Sanderson ${ }^{[18]}$ experimentally and analytically examined the effects of nonequilibrium on type IV flows. A real gas numerical study was done by Brück ${ }^{[19]}$ which looked at the effects of nonequilibrium and impinging shock location on flow structure and surface pressure and heat flux. These real gas studies, however, have only addressed the issue of steady flows. Though ideal gas shock interference has been found to be unsteady and the unsteadiness has strong effects on surface heating rates, so far, no work in studying real gas effects in unsteady shock interactions, such as those around a maneuvering hypersonic vehicle, has been done, to the authors' knowledge.

Therefore, the objective of our work is to study viscous and nonequilibrium effects in steady shock wave reflections and unsteady type IV shock interference heating problems using numerical simulations. The present paper focuses on work done in studying the fluid mechanics and real gas effects in unsteady viscous shock reflection and interaction flow fields.
Specifically, we have numerically studied the effects of thermo-chemical nonequilibrium on the flow characteristics by using currently available thermal and chemical nonequilibrium models.

The viscous flows are modeled by the multicomponent Navier-Stokes equations with a three temperature model and nonequilibrium rotational, vibrational, and chemical modes. Though rotational nonequilibrium is only important in the low density regime, it is included in the present model for use in planned future studies at lower densities. At present, air flow in the temperature regime below $8000-9000^{\circ} \mathrm{K}$ is considered. This allows ionization of the gas to be neglected for simplicity. The models follow those of Candler ${ }^{[21]}$, Gökçen ${ }^{[22]}$, and Park ${ }^{[23]}$. The numerical method is a secondorder TVD scheme with Roe's approximate Riemann solver as extended to nonequilibrium flows by Grossman and Cinella ${ }^{[25]}$. The source terms are computed using the Additive Semi-implicit RungeKutta method derived by Zhong ${ }^{[26]}$.

\section{Physical Model}

\section{Equations of Motion}

In conservative form, the multicomponent NavierStokes Equations, along with the rotational and vibrational energy equations, are:

$$
\begin{gathered}
\frac{\partial \rho_{i}}{\partial t}+\frac{\partial}{\partial x}\left(\rho_{i} u+j_{i x}\right)+\frac{\partial}{\partial y}\left(\rho_{i} v+j_{i y}\right)=w_{i} \\
\frac{\partial(\rho u)}{\partial t}+\frac{\partial}{\partial x}\left(\rho u^{2}+p-\tau_{x x}\right)+\frac{\partial}{\partial y}\left(\rho u v-\tau_{x y}\right)=0 \\
\frac{\partial(\rho u)}{\partial t}+\frac{\partial}{\partial x}\left(\rho u v-\tau_{x y}\right)+\frac{\partial}{\partial y}\left(\rho v^{2}+p-\tau_{y y}\right)=0 \\
\frac{\partial E_{r}}{\partial t}+\frac{\partial}{\partial x}\left(u E_{r}+q_{r x}\right)+\frac{\partial}{\partial y}\left(v E_{r}+q_{r y}\right)=w_{r} \\
\frac{\partial E_{v}}{\partial t}+\frac{\partial}{\partial x}\left(u E_{v}+q_{v x}\right)+\frac{\partial}{\partial y}\left(v E_{v}+q_{v y}\right)=w_{v} \\
\frac{\partial E}{\partial t}+\frac{\partial}{\partial x}\left[u(E+p)-u \tau_{x x}-v \tau_{x y}+q_{x}\right] \\
+\frac{\partial}{\partial y}\left[v(E+p)-u \tau_{x y}-v \tau_{y y}+q_{y}\right]=0
\end{gathered}
$$

where $E_{r}, E_{v}$, and $E$ are the rotational, vibrational, and total energies per unit volume, respectively, the 
$w_{i}$ 's are the chemical source terms for species $i$, and $w_{r}$ and $w_{v}$ are the source terms for the rotational and vibrational modes respectively.

\section{Equations of State}

The system is taken to be a mixture of thermally perfect gases with the following equation of state:

$$
p=\rho R T_{t}
$$

where $p$ and $\rho$ are the sums of the species pressures and densities, respectively, and $R$ is the mass averaged gas constant, defined as:

$$
R=\sum \frac{\rho_{i}}{\rho} R_{i}
$$

$R_{i}$ is the species specific gas constant, and $T_{t}$ is the translational temperature.

The nonequilibrium rotational and vibrational energy modes are modeled by separate temperatures, $T_{r}$ and $T_{v}$, respectively $[21,22,27]$. This leads to the following internal energy equations:

For diatomic species:

$$
\begin{gathered}
e_{i}=\frac{3}{2} R_{i} T_{t}+e_{r i}+e_{v i}+h_{i}^{\circ} \\
e_{r i}=R_{i} T_{r} \\
e_{v i}=R_{i} \frac{\theta_{v i}}{e^{\theta_{v i} / T_{v}}-1}
\end{gathered}
$$

where $\theta_{v i}$ is the characteristic vibrational temperature of species $i$.

For monatomic species:

$$
e_{i}=\frac{3}{2} R_{i} T_{t}+h_{i}^{\circ}
$$

This leads to the bulk energy equations:

$$
\begin{gathered}
E_{r}=\sum \rho_{i} e_{r i} \\
E_{v}=\sum \rho_{i} e_{v i} \\
E=\sum \rho_{i} e_{i}+\rho \frac{u^{2}+v^{2}}{2}
\end{gathered}
$$

\section{Constitutive Relations}

The viscous stresses are modeled using the NavierStoke equations:

$$
\begin{gathered}
\tau_{x x}=\frac{2}{3} \mu\left[2 \frac{\partial u}{\partial x}-\frac{\partial v}{\partial y}\right] \\
\tau_{x y}=\mu\left[\frac{\partial u}{\partial y}+\frac{\partial v}{\partial x}\right] \\
\tau_{y y}=\frac{2}{3} \mu\left[2 \frac{\partial v}{\partial y}-\frac{\partial u}{\partial x}\right]
\end{gathered}
$$

For chemically reacting flow, multicomponent diffusion is approximately modeled by Fick's Law for binary diffusion:

$$
\begin{aligned}
& j_{i x}=-\rho D \frac{\partial\left(\rho_{i} / \rho\right)}{\partial x} \\
& j_{i y}=-\rho D \frac{\partial\left(\rho_{i} / \rho\right)}{\partial y}
\end{aligned}
$$

Diffusion due to thermal and pressure gradients can be neglected for the temperature regime currently being studied $\left(<9000^{\circ} \mathrm{K}\right)$.

Heat diffusion is modeled using Fourier's Law for heat conduction:

$$
\begin{aligned}
& q_{x}=-\kappa_{t} \frac{\partial T_{t}}{\partial x}-\kappa_{r} \frac{\partial T_{r}}{\partial x}-\kappa_{v} \frac{\partial T_{v}}{\partial x}+\sum j_{i x} h_{i} \\
& q_{y}=-\kappa_{t} \frac{\partial T_{t}}{\partial y}-\kappa_{r} \frac{\partial T_{r}}{\partial y}-\kappa_{v} \frac{\partial T_{v}}{\partial y}+\sum j_{i y} h_{i}
\end{aligned}
$$

The transport coefficients need to be modeled for a gas mixture and, where appropriate, for the nonequilibrium energy modes. Individual species viscosities $\left(\mu_{i}\right)$ are calculated using a curve fit model presented by Moss ${ }^{[28]}$ :

$$
\mu_{i}=\exp \left[\left(A_{i} \ln T_{t}+B_{i}\right) \ln T_{t}+C_{i}\right]
$$

where $A_{i}, B_{i}$, and $C_{i}$ are tabulated empirical constants.

The viscosity of the mixture is then found from Wilke's formulation ${ }^{[29]}$ :

$$
\begin{gathered}
\mu=\sum_{i} \frac{X_{i} \mu_{i}}{\sum_{j} X_{i} \phi_{i j}} \\
\phi_{i j}=\frac{\left[1+\left(\frac{\mu_{i}}{\mu_{j}}\right)^{\frac{1}{2}}\left(\frac{\mathcal{M}_{j}}{\mathcal{M}_{i}}\right)^{\frac{1}{4}}\right]^{2}}{\left[8\left(1+\frac{\mathcal{M}_{i}}{\mathcal{M}_{j}}\right)\right]^{\frac{1}{2}}}
\end{gathered}
$$


where $\mathcal{M}_{i}$ is the molecular weight of species $i$, and $X_{i}$ is the mole fraction of species $i$.

The binary diffusion coefficient, $D$, is found by assuming a constant Schmidt number ${ }^{[22]}$ :

$$
S c=\frac{\mu}{\rho D}=0.5
$$

The individual species translational heat conduction coefficients $\left(\kappa_{t i}\right)$ are given by Euken's relation [27]:

$$
\kappa_{t i}=\frac{5}{2} \mu_{i} c_{v t i}
$$

where

$$
c_{v t i}=\frac{3}{2} R_{i}
$$

The overall coefficient is then calculated using Wilke's formula in a similar fashion to the viscosity coefficient ${ }^{[20]}$.

The thermal conductivities associated with the rotational and translational modes are given by ${ }^{[22]}$ :

$$
\begin{aligned}
& \kappa_{r}=\sum \mu_{i} c_{v r i} \\
& \kappa_{v}=\sum \mu_{i} c_{v v i}
\end{aligned}
$$

where the sums are taken over the diatomic species only and:

$$
\begin{gathered}
c_{v r i}=R_{i} \\
c_{v v i}=\frac{R_{i}\left(\theta_{v i} / T_{v}\right)^{2} e^{\theta_{v i} / T_{v}}}{\left(e^{\theta_{v i} / T_{v}}\right)^{2}}
\end{gathered}
$$

\section{Source Terms}

Chemistry is modeled using a five species model $\left(N_{2}, O_{2}, N O, O, N\right)$ without ionization. For the test cases considered in this paper, the temperatures are not expected to exceed $9000 \mathrm{~K}$, which is the threshold for ionization ${ }^{[20]}$. The five species reaction model is given as:

$$
\begin{aligned}
N_{2}+M_{i} & \rightleftharpoons N+N+M_{i} \\
O_{2}+M_{i} & \rightleftharpoons O+O+M_{i} \\
N O+M_{i} & \rightleftharpoons N+O+M_{i} \\
N_{2}+O & \rightleftharpoons N O+N \\
N O+O & \rightleftharpoons O_{2}+N
\end{aligned}
$$

where $M_{i}$ denotes any of the $i$ species. Rates for each of these 17 reactions can written as:

$$
\begin{aligned}
\mathcal{R}_{1} & =\sum_{i} k_{b 1 i}\left[N_{2}\right]\left[M_{i}\right]-k_{f 1 i}[N]^{2}\left[M_{i}\right] \\
\mathcal{R}_{2} & =\sum_{i} k_{b 2 i}\left[O_{2}\right]\left[M_{i}\right]-k_{f 2 i}[O]^{2}\left[M_{i}\right] \\
\mathcal{R}_{3} & \left.=\sum_{i} k_{b 3 i}[N O]\left[M_{i}\right]-k_{f 3 i}[N][O]\left[M_{i}\right] 40\right) \\
\mathcal{R}_{4} & =k_{b 4}\left[N_{2}\right][O]-k_{f 4}[N O][N] \\
\mathcal{R}_{5} & =k_{b 5}[N O][O]-k_{f 5}\left[O_{2}\right][N]
\end{aligned}
$$

The rate coefficients (the $k_{f}$ and $k_{b}$ 's) used are the Dunn-Kang rate coefficients based on a two temperature model as presented by Häuser, et al. ${ }^{[24]}$. With these rate coefficients, expressions for the species source terms can be written:

$$
\begin{aligned}
& w_{1}=\mathcal{M}_{1}\left(\mathcal{R}_{1}+\mathcal{R}_{4}\right) \\
& w_{2}=\mathcal{M}_{2}\left(\mathcal{R}_{2}-\mathcal{R}_{5}\right) \\
& w_{3}=\mathcal{M}_{3}\left(\mathcal{R}_{3}-\mathcal{R}_{4}+\mathcal{R}_{5}\right) \\
& w_{4}=\mathcal{M}_{4}\left(-2 \mathcal{R}_{1}-\mathcal{R}_{3}-\mathcal{R}_{4}-\mathcal{R}_{5}\right) \\
& w_{5}=\mathcal{M}_{5}\left(-2 \mathcal{R}_{2}-\mathcal{R}_{3}+\mathcal{R}_{4}+\mathcal{R}_{5}\right)
\end{aligned}
$$

The energy transfer modes considered are translation-vibration coupling, vibrationdissociation coupling, and rotation-translation coupling. Other modes were assumed to be negligible in their effect ${ }^{[22]}$. Rotation-dissociation coupling was omitted as the authors are unaware of a suitable, accepted model for this. Vibration-translation and rotation-translation modes were modeled using the Landau-Teller model, which requires and expression for the respective relaxation time associated with the transfer mode.

The expression for vibration-translation coupling is:

$$
Q_{T-V}=\sum_{j} \frac{\rho_{j} R_{j} \theta_{v j}\left(\frac{1}{e^{\theta_{v j} / T_{t}}-1}-\frac{1}{e^{\theta_{v j} / T_{v}}-1}\right)}{\tau_{v j}}
$$

where the sum is taken over the diatomic species only.

For the vibrational relaxation time of species $j$, $\tau_{v j}$, the corrected Millikan and White formula as proposed by Park ${ }^{[30,23]}$ was used. This gives the vibrational time as:

$$
\tau_{v j}=<\tau_{v j}>+\tau_{c j}
$$


with

$$
<\tau_{v j}>=\frac{\sum_{i} X_{i}}{\sum_{i} X_{i} / \tau_{v i j}^{M W}}
$$

(where the sums are taken over all species)

$$
\begin{gathered}
\tau_{v i j}^{M W}=\frac{1}{p} \exp \left[A \mu_{i j}^{-\frac{1}{2}} \theta_{v j}^{\frac{4}{3}}\left(T_{t}^{-\frac{1}{3}}-B \mu_{i j}^{\frac{1}{4}}\right)-C\right] \\
A=1.16 \times 10^{-3} \\
B=0.015 \\
C=18.42
\end{gathered}
$$

and

$$
\tau_{c j}=\frac{1}{\bar{c}_{j} \sigma_{v} N}
$$

where $X_{i}$ is the mole fraction of species $i, p$ is in atmospheres, $\theta_{v j}$ is the characteristic vibrational temperature of species $j, \mu_{i j}$ is the reduced mass given by

$$
\mu_{i j}=\frac{\mathcal{M}_{i} \mathcal{M}_{j}}{\mathcal{M}_{j}+\mathcal{M}_{i}},
$$

$\bar{c}_{j}$ is the mean molecular speed given by

$$
\bar{c}_{j}=\sqrt{\frac{8 R_{j} T_{t}}{\pi}},
$$

$\sigma_{v}$ is the limited collision cross section (in $m^{2}$ ) given by ${ }^{[31,32]}$

$$
\sigma_{v}=10^{-21}\left(\frac{50,000}{T_{t}}\right)^{2}
$$

and $N$ is the total number density of the gas.

The model used for vibration-dissociation coupling is the one employed by Candler in Ref.[21] and is given by

$$
Q_{V-D}=\sum_{j} w_{j} \frac{R_{j} \theta_{v j}}{e^{\theta_{v j} / T_{v}}-1}
$$

where the sum is taken over the diatomic species only.

The vibrational source term is then:

$$
w_{v}=Q_{T-V}+Q_{V-D}
$$

The rotational-translational term is given by:

$$
w_{r}=Q_{T-R}=\sum_{j} \frac{\rho_{j} R_{j}\left(T_{t}-T_{r}\right)}{\tau_{r j}}
$$

where the sum is taken over the diatomic species only.

For rotation-translation coupling, the relaxation time, $\tau_{r i}$ was calculated using the formulation suggested by Gökçen [22] where the relaxation time is calculated by multiplying the molecular collision time by a characteristic collision number. For this study, the number used was 5 , following what was done by Gökçen ${ }^{[22,33]}$. This leads to:

$$
\tau_{r j}=\frac{5}{\sqrt{8 \pi R_{j} T_{t}} \sum_{i} N_{i} d_{j i}^{2} \sqrt{1+\mathcal{M}_{j} / \mathcal{M}_{i}}}
$$

where $N_{i}$ is the number density of species $i, d_{j i}$ is the average collision diameter between species $j$ and $i$, and the sum is taken over all species.

\section{Numerical Method}

The unsteady flow field is solved using a second order TVD scheme, while the source terms will be computed via a semi-implicit Runge-Kutta method [26]. The conservation laws were discretized using a finite volume formulation.

$$
\frac{\partial \mathbf{U}}{\partial t}+\frac{\partial}{\partial x}\left(\mathbf{F}+\mathbf{F}_{\mathbf{v}}\right)+\frac{\partial}{\partial y}\left(\mathbf{G}+\mathbf{G}_{\mathbf{v}}\right)=\mathbf{W}
$$

where the conserved quantity and source term vectors are:

$$
\mathbf{U}=\left[\begin{array}{c}
\rho_{1} \\
\rho_{2} \\
\rho_{3} \\
\rho_{4} \\
\rho_{5} \\
\rho u \\
\rho v \\
E_{r} \\
E_{v} \\
E
\end{array}\right], \quad \mathbf{W}=\left[\begin{array}{c}
w_{1} \\
w_{2} \\
w_{3} \\
w_{4} \\
w_{5} \\
0 \\
0 \\
w_{r} \\
w_{v} \\
0
\end{array}\right]
$$


Inviscid fluxes:

$$
\mathbf{F}=\left[\begin{array}{c}
\rho_{1} u \\
\rho_{2} u \\
\rho_{3} u \\
\rho_{4} u \\
\rho_{5} u \\
\rho u^{2}+p \\
\rho v u \\
u E_{r} \\
u E_{v} \\
u(E+p)
\end{array}\right], \mathbf{G}=\left[\begin{array}{c}
\rho_{1} v \\
\rho_{2} v \\
\rho_{3} v \\
\rho_{4} v \\
\rho_{5} v \\
\rho u v \\
\rho v^{2}+p \\
v E_{r} \\
v E_{v} \\
v(E+p)
\end{array}\right]
$$

Diffusive fluxes:

$$
\mathbf{F}_{\mathbf{v}}=\left[\begin{array}{c}
j_{1 x} \\
j_{2 x} \\
j_{3 x} \\
j_{4 x} \\
j_{5 x} \\
-\tau_{x x} \\
-\tau_{x y} \\
q_{r x} \\
q_{v x} \\
Q_{x}
\end{array}\right], \quad \mathbf{G}_{\mathbf{v}}=\left[\begin{array}{c}
j_{1 y} \\
j_{2 y} \\
j_{3 y} \\
j_{4 y} \\
j_{5 y} \\
-\tau_{x y} \\
-\tau_{y y} \\
q_{r y} \\
q_{v y} \\
Q_{y}
\end{array}\right]
$$

where $Q_{x}=-u \tau_{x x}-v \tau_{x y}+q_{x}$ and $Q_{y}=-u \tau_{x y}-$ $v \tau_{y y}+q_{y}$.

The conservation laws were then cast into integral form in terms of cell averages so that the finite volume technique could be applied:

$$
\frac{\partial \mathbf{U}_{i j}}{\partial t}+\frac{1}{V_{i j}} \oint_{S} \mathbf{E} \cdot d \mathbf{s}=\mathbf{W}_{i j}
$$

where

$$
\mathbf{E}=\left(\mathbf{F}+\mathbf{F}_{\mathbf{v}}\right) \hat{\imath}+\left(\mathbf{G}+\mathbf{G}_{\mathbf{v}}\right) \hat{\jmath}
$$

This expression "integrates" for each $i j$ cell to:

$$
\begin{gathered}
\frac{\partial \mathbf{U}_{i j}}{\partial t}+\frac{1}{V_{i j}}\left[(\mathbf{E} \cdot \mathbf{S})_{i+\frac{1}{2}, j}-(\mathbf{E} \cdot \mathbf{S})_{i-\frac{1}{2}, j}\right. \\
\left.+(\mathbf{E} \cdot \mathbf{S})_{i, j+\frac{1}{2}}-(\mathbf{E} \cdot \mathbf{S})_{i, j-\frac{1}{2}}\right]=\mathbf{W}_{i j}
\end{gathered}
$$

The inviscid fluxes were solved via a 2nd order TVD formulation using characteristic variable extrapolation with the Roe flux difference splitting Riemann solver ${ }^{[34,35]}$ and the minmod limiter. An inviscid flux vector $\mathbf{F}$ may be expressed in terms of its Jacobian and the conservative variable vector $\mathrm{U}$ :

$$
\mathbf{F}=\frac{\partial \mathbf{F}}{\partial \mathbf{U}} \mathbf{U}=\mathbf{A} \cdot \mathbf{U}
$$

A can then be expressed in terms of $\Lambda$, the diagonal matrix of its eigenvalues, and $\mathbf{T}^{-1}$ and $\mathbf{T}$, the eigenvector matrices, as $\mathbf{A}=\mathrm{T}^{-1} \Lambda \mathrm{T}$.

In the Roe schemes, the cell-face fluxes for the flux vector $\mathbf{F}$ at the cell face $i+1 / 2$ may be expressed as [36]:

$\mathbf{F}_{i+1 / 2}=\frac{1}{2}\left[\mathbf{F}\left(\mathbf{U}^{R}\right)+\mathbf{F}\left(\mathbf{U}^{L}\right)\right]-\frac{1}{2} \hat{\mathbf{T}}^{-1}|\hat{\mathbf{\Lambda}}| \hat{\mathbf{T}}\left(\mathbf{U}^{R}-\mathbf{U}^{L}\right)$

The $\left({ }^{\wedge}\right)$ indicates the quantity is evaluated using the Roe averages at the $i+1 / 2$ face, which were based on the formulations given by Grossman, et al. $[25,35]$ for nonequilibrium gases. $\mathrm{U}^{R}$ and $\mathrm{U}^{L}$ are calculated from the characteristic variables, $\mathcal{W}^{R}, \mathcal{W}^{L}$, which are extrapolated to the cell faces using the minmod limiter. The characteristic variables are related to the conservative variables by:

$$
\mathcal{W}=\mathbf{Q U}
$$

The left and right states at a given cell face are calculated using a slope limiter ${ }^{[37]}$. For cell face $i+1 / 2$, the right state is:

$$
\mathcal{W}^{R}=\mathcal{W}_{i+1}-\frac{1}{2} \operatorname{minmod}\left[\Delta_{i+1}, \Delta_{i}\right]
$$

where:

$$
\begin{aligned}
\mathcal{W}_{i+1} & =\mathbf{Q}_{i+1} \mathrm{U}_{i+1}, \\
\mathcal{W}_{i+2} & =\mathbf{Q}_{i+1} \mathrm{U}_{i+2}, \\
\mathcal{W}_{i} & =\mathbf{Q}_{i+1} \mathrm{U}_{i} \\
\Delta_{i} & =\mathcal{W}_{i+1}-\mathcal{W}_{i}
\end{aligned}
$$

The left is given by:

$$
\mathcal{W}^{L}=\mathcal{W}_{i}-\frac{1}{2} \operatorname{minmod}\left[\Delta_{i}, \Delta_{i-1}\right]
$$

where:

$$
\begin{aligned}
\mathcal{W}_{i} & =\mathbf{Q}_{i} \mathrm{U}_{i}, \\
\mathcal{W}_{i+1} & =\mathbf{Q}_{i} \mathrm{U}_{i+1}, \\
\mathcal{W}_{i-1} & =\mathbf{Q}_{i} \mathrm{U}_{i-1}, \\
\Delta_{i} & =\underline{W}_{i \neq 1}-\mathcal{W}_{i}
\end{aligned}
$$

The minmod function is defined here as ${ }^{[37]}$ :

$\min \bmod (a, b)=\frac{1}{2}[\operatorname{sgn}(a)+\operatorname{sgn}(b)] \min (|a|,|b|)$ 
From here, the left and right conservative variable vectors, $\mathrm{U}^{R}=\mathbf{Q}_{i+1}^{-1} \mathcal{W}^{R}$ and $\mathbf{U}^{L}=\mathbf{Q}_{i}^{-1} \mathcal{W}^{L}$ respectively, can be computed for the $i+1 / 2$ face.

Viscous fluxes were discretized via central differences. Time stepping was carried out using second and third-order Runge-Kutta methods. Explicit time integration is suitable for hypersonic viscous flow with relatively low density and/or temperature where the source terms are not expected to be overly stiff, as is the case for perfect gas flow. For nonequilibrium calculations, the source terms are numerically too stiff to handle explicitly. In this case, a class of semi-implicit Runge-Kutta schemes developed by Zhong ${ }^{[26]}$ were employed.

The third-order Additive Semi-Implicit RungeKutta scheme that was employed can be expressed as:

$$
\begin{array}{r}
{\left[\mathbf{I}-h a_{1} \mathbf{J}\left(\mathbf{U}^{n}\right)\right] \mathbf{k}_{1}=h\left[\mathbf{F}\left(\mathbf{U}^{n}\right)+\mathbf{G}\left(\mathbf{U}^{n}\right)\right]} \\
{\left[\mathbf{I}-h a_{2} \mathbf{J}\left(\mathbf{U}^{n}+c_{21} \mathbf{k}_{1}\right)\right] \mathbf{k}_{2}=} \\
h\left[\mathbf{F}\left(\mathbf{U}^{n}+b_{21} \mathbf{k}_{1}\right)+\mathbf{G}\left(\mathbf{U}^{n}+c_{21} \mathbf{k}_{1}\right)\right] \\
{\left[\mathbf{I}-h a_{3} \mathbf{J}\left(\mathbf{U}^{n}+c_{31} \mathbf{k}_{1}+c_{32} \mathbf{k}_{2}\right)\right] \mathbf{k}_{3}=} \\
h\left[\mathbf{F}\left(\mathbf{U}^{n}+b_{31} \mathbf{k}_{1}+b_{32} \mathbf{k}_{2}\right)\right. \\
\left.\left.+\mathbf{G}\left(\mathbf{U}^{n}+c_{31} \mathbf{k}_{1}\right)+c_{32} \mathbf{k}_{2}\right)\right] \\
\mathbf{U}^{n+1}=\mathbf{U}^{n}+\omega_{1} \mathbf{k}_{1}+\omega_{2} \mathbf{k}_{2}+\omega_{3} \mathbf{k}_{3}
\end{array}
$$

where $U^{n}$ is the vector of conserved quantities at step $n, \mathbf{F}$ is the conservative flux vector, $\mathbf{G}$ is the stiff source term vector, $\mathbf{J}=\frac{\partial \mathbf{G}}{\partial \mathrm{U}}$ is the Jacobian matrix of the source term, and $h$ is the timestep. The coefficients are ${ }^{[26]}$ :

$$
\begin{aligned}
\omega_{1} & =1 / 8 & \omega_{2} & =1 / 8 \\
\omega_{3} & =3 / 4 & b_{21} & =8 / 7 \\
b_{31} & =71 / 252 & b_{32} & =7 / 36 \\
a_{1} & =0.797097 & a_{2} & =0.591381 \\
a_{3} & =0.134705 & c_{21} & =1.05893 \\
c_{31} & =1 / 2 & c_{32} & =-0.375939
\end{aligned}
$$

\section{Validation Results}

Pseudo-Steady Mach Reflection

A perfect gas pseudo-steady Mach reflection case was studied and compared with available experimental and numerical results ${ }^{[6,3,7]}$ to validate the time accurate capabilities of the code. A schematic of the Mach reflection flow field is presented in Figure 5 which illustrates the Mach stem and slip line characteristic of this flow. This type of flow, under ideal conditions (inviscid, equilibrium flow) is called pseudo steady because the resulting shock/slip line patterns were found to be self-similar, at least with respect to the configuration of the discontinuities ${ }^{[2]}$. The test case involved a Mach 2.03 shock wave hitting a $27^{\circ}$ wedge in quiescent air at a density of $0.387 \mathrm{~kg} / \mathrm{m}^{3}$ and a pressure of $33.3 \times 10^{3} \mathrm{~Pa}$. For this case, an algebraically generated 300 by 300 grid was used, an example of which is shown in Figure 6.

Instantaneous density contours for this flow are shown in Figure 8, pressure contours are presented in Figure 9, and temperature contours in Figure 10. The incident shock, reflected shock, and Mach stem are evident in all three contours. The slip line does not appear in the pressure contour as it is a contact discontinuity and not a shock. Density, normalized by the freestream density, profiles at $(\mathrm{J}=2)$ and 3 computational cells $(\mathrm{J}=5)$ away from the wall are shown in Figure 7. The $\mathrm{x}$-axis has been normalized by the distance from the ramp corner to the Mach stem, with the Mach stem located at $X / L=0$. Good qualitative agreement between the interferograms of Glaz, et. al. ${ }^{[7]}$ and our density contours was obtained. Also, good quantitative agreement between computed density profiles and those presented by Deschambault ${ }^{[6]}$ as shown in Figure 7. However, there was some discrepancy, especially in the region adjacent to the wall between the Mach stem and the slip line, which corresponds to $X / L=0.25$ to 0.4 in Figure 7. There were two sources for this discrepancy. First, the grid was algebraically generated with the vertical grid lines aligned with the incident normal shock. As a consequence, the grid was misaligned by as much as $27^{\circ}$ relative to the Mach stem (assuming it is perpendicular to the wall). The misalignment of the grid and the strong discontinuity (Mach stem) could have led to resolution difficulties near the wall-Mach stem junction. Second, the experimental density values ${ }^{[6]}$ were derived from interferometric data. However, the state behind the Mach stem was calculated from shock jump relations, rather than from interferometric data due to resolution difficulties ${ }^{[38]}$. The relations from three shock theory hold near the triple point,-and would be valid all the was down to the wall if uniform flow in the region behind the Mach stem were assumed. However, the flow there is not uniform, due to the turning needed for a consistent streamline structure. The fact that the difference between computation 
and theory was related to the conditions near the wall is apparent as the discrepancy diminishes if density levels just 3 cells removed from the wall are examined (see Figure 7).

\section{Steady Flow Past a Cylinder}

As a test case, hypervelocity flow past a cylinder based on an experiment by Hornung [39] was computed using the current model. The case was flow past a 1 inch diameter cylinder with $u_{\infty}=5590 \mathrm{~m} / \mathrm{s}, T_{\infty}=1833 \mathrm{~K}, p_{\infty}=2910 \mathrm{~Pa}$ and $\mathrm{a}$ Reynolds number of 6000 . The gas in the freestream was partially dissociated nitrogen, $92.7 \% \mathrm{~N}_{2}$ and $7.3 \% N$ by mass and conditions match those studied experimentally by Hornung and computationally by Candler ${ }^{[40]}$. Computed density contours are compared with an experimental interferogram of Hornung ${ }^{[39]}$ in Figure 4. The current three temperature model yields density contours which match well with experiment. The computed shock shape and stand off distance agrees very well with the shock structure shown in the interferogram.

\section{Steady Shock-Boundary Layer Interaction with Real Gas Effects}

A steady shock-boundary layer interaction was studied for hypersonic flow with nonequilibrium effects. A schematic of this flow field is given in Figure 2. The flow deflection angle was $22^{\circ}$, the frozen flow freestream Mach number was 7.0, the gas was air with a freestream composition of $79 \% N_{2}$ and $21 \%$ $\mathrm{O}_{2}$ by mass, and the Reynolds Number, based on freestream values and the shock impingement distance from the leading edge, was $3.571 \times 10^{5}$. The shock impingement distance from the leading edge, based on a geometric calculation, was $0.2134 m$. The incident shock was generated by setting the desired flow deflection at the upper boundary condition and a no slip, $1200^{\circ} \mathrm{K}$ isothermal wall boundary condition was used at the plate surface. An 88 by 72 ( $x$ by $y$ ) Cartesian grid, exponentially stretched in the $y$ direction, was used in these calculations. The simulations were run to steady-state at a CFL number of 0.3 .
Effects of Thermal and Chemical Nonequilibrium

Cases were run with for perfect (ideal), vibrationally excited but chemically frozen, and nonequilibrium (reacting) models with a freestream temperature of $1600^{\circ} \mathrm{K}$ and a freestream density of $0.0169 \mathrm{~kg} / \mathrm{m}^{3}$. First, comparisons were made between the three models to examine the impact of the presence of real gas effects. The differences in flow structure are demonstrated in the translational temperature and Mach number contours for the three models presented in Figure 15. The contours show the region around the shock impingement region, and the $y$-axis has been stretched by a factor of 10 for clarity. In each plot, the incident shock can be seen impinging on the wall. Ahead of the shock impingement point in each figure is a separation bubble. The reflected shock can be seen in each picture where the separated flow reattaches behind the separation bubble. It can be seen by comparing the temperature levels in the ideal (Figure 16a), frozen (Figure $16 \mathrm{~b}$ ), and reacting (Figure 16c) contours that the peak temperature in the shock impingement region were highest for the ideal case and lowest for the reacting case. This is more clearly demonstrated by the surface temperature profiles shown in Figure 14 where the decrease in temperature between the three models can be clearly seen.

Another effect is the reduction in size of the separation bubble which can been seen by comparing the Mach number contours. The separation region is largest in Figure 15a, decreases in size in Figure $15 \mathrm{~b}$, and is smallest in Figure 15c. To quantify this change, bubble size was nondimensionalized using the distance from the leading edge the shock would impinge at for the perfect inviscid gas case. The effects on separation bubble size were as follows:

\begin{tabular}{|l|l|}
\hline Model & Bubble Size \\
\hline Ideal Gas & 0.4920 \\
\hline Vibration Only & 0.4218 \\
\hline Real Gas & 0.3046 \\
\hline
\end{tabular}

This is illustrated in the comparison of skin friction coefficient profiles in Figure 11 where the separation region, taken as the region where $c_{f}$ is negative, can been seen to decrease in size between the three models.

The decrease in temperature and bubble size was due to the endothermic nonequilibrium effects. These effects were also seen in the surface pressure 
profiles, shown in Figure 12, where the ideal case exhibited the largest pressure jump, while the reacting case showed the lowest jump, and in the surface heat transfer profiles given in Figure 13, where the the ideal case resulted in the highest heating rate to the wall while the lowest heating rate was given by the reacting case. Between the ideal and frozen cases, the energy is distributed among more internal modes in the frozen case, resulting in a lower thermodynamic temperature in that case. As for the large difference between the frozen and reacting cases, dissociation reactions are endothermic, absorbing energy from the flow to break the chemical bonds. This reduces the temperature of the gas, and, consequently, the pressure, as can be seen in the pressure profiles in Figure 12. This results in a less severe adverse pressure gradient and a smaller separation bubble and lower heat transfer rates to the wall, which are shown in Figure 13.

\section{Effects of Freestream Enthalpy}

Next, the effect of freestream enthalpy on the reacting model was studied using the following conditions:

\begin{tabular}{|l|l|l|}
\hline Case & $T_{\infty}$ & $\rho_{\infty}$ \\
\hline 1 & $700 K$ & $0.0140 \mathrm{~kg} / \mathrm{m}^{3}$ \\
\hline 2 & $1200 \mathrm{~K}$ & $0.0158 \mathrm{~kg} / \mathrm{m}^{3}$ \\
\hline 3 & $1600 \mathrm{~K}$ & $0.0169 \mathrm{~kg} / \mathrm{m}^{3}$ \\
\hline 4 & $2000 \mathrm{~K}$ & $0.0178 \mathrm{~kg} / \mathrm{m}^{3}$ \\
\hline
\end{tabular}

Contours are plotted for Mach number in Figures $15 \mathrm{c}, 17 \mathrm{a}, 17 \mathrm{~b}$, and $17 \mathrm{c}$ and for translational temperature in Figures 16c,18a, 18b, 18c. The degree of dissociation can be seen in the surface mass fraction profiles in Figures 19a, 19b, and 19c. The point at which dissociation begins in these profiles is coincident with the front edge of the separation bubble due to the leading edge shock that forms at the boundary layer detachment point. This occurs at $X=0.12$ for Case 2, as seen by comparing Figures $18 \mathrm{~b}$ and $19 \mathrm{a}$, and at $X=0.19$ for Cases 3 and 4 , as seen by comparing Figure $16 \mathrm{c}$ with $19 \mathrm{~b}$ and $18 \mathrm{c}$ with 19c. As the freestream temperature increases, the separation bubble can be seen to decrease in size by examining the Mach number contours in sequence in Figures $17 \mathrm{a}, 17 \mathrm{~b}$, and $15 \mathrm{c}$. However, for the highest temperature case, Case 4 , this trend reverses. This is best illustrated by examining the skin friction coefficient profiles in Figure 20. By normalizing the separation bubble size as done previously, this trend can be quantified as follows:

\begin{tabular}{|l|l|l|}
\hline Case & $T_{\infty}$ & Bubble Size \\
\hline 1 & $700 K$ & 0.539 \\
\hline 2 & $1200 K$ & 0.422 \\
\hline 3 & $1600 K$ & 0.305 \\
\hline 4 & $2000 K$ & 0.374 \\
\hline
\end{tabular}

A point to note is that there was a difference in flow structure between Case 4 and the other three cases, which can be seen by examining the surface pressure profiles in Figure 21 and the surface heat transfer profiles in Figure 22 and noting the distinct differences in profile shapes between Case 4 $(2000 \mathrm{~K})$ and the other three cases. This was due to the fact that $N_{2}$ has a higher dissociation temperature than $\mathrm{O}_{2}$. Is Figures $19 \mathrm{a}$ and $19 \mathrm{~b}$, no significant amounts of atomic nitrogen were observed. However, in the $T=2000^{\circ} \mathrm{K}$ case, temperatures became high enough so that nitrogen dissociation occurred in measurable quantities, as seen at $X=0.3$ in Figure $19 \mathrm{c}$, and changed the flow field. This nitrogen dissociation region correspond to where the pressure profile for Case 4 in Figure 21 departs from the trends of the other three cases. Instead of of a pressure drop as the oxygen recombines to equilibrium values, the pressure for the $T=2000^{\circ} \mathrm{K}$ case continues to increase, extending the separation region.

The results we have obtained here indicate that real gas effects significantly influence the structure of hypervelocity shock/boundary layer interactions. In this study, the presence of real gas effects decreased the size of the shock induced separation region relative to the perfect gas case. Also, the post impingement surface heating rates were significantly reduced by the endothermic relaxation of the internal and chemical modes present in the real gas. The direction of this influence, however, is dependent on the nature of the nonequilibrium. Earlier work done by Ballaro and Anderson ${ }^{[4]}$, and Grumet, et al. ${ }^{[5]}$ indicated that real gas effects could increase the heating rates. However, in those studies, the dominating reactions near the wall were recombination reactions, which are exothermic, while in the present case, the dominating reactions were dissociation reactions, which are endothermic. 
Unsteady Type IV Shock Interference Heating with Real Gas Effects

Here, a type IV problem with real gas and unsteady effects was studied. The case was based on that studied in Refs. [11] and [15]. In order to study the effect of thermochemical nonequilibrium, the original free stream conditions were altered so that dissociation would occur behind the bow shock. The new freestream was an undissociated $N_{2}$ flow with the following conditions:

\begin{tabular}{|c|c|}
\hline Cylinder Radius & $0.0381 \mathrm{~m}$ \\
\hline $\begin{array}{c}\text { Flow Deflection } \\
\text { (impinging shock) }\end{array}$ & $12^{\circ}$ \\
\hline$R e$ & $2.57354 \times 10^{5}$ \\
\hline$M_{\infty}$ & 8.03 \\
\hline$P_{\infty}$ & $985.015 \mathrm{~Pa}$ \\
\hline$T_{\infty}$ & $800 \mathrm{~K}$ \\
\hline$T_{\text {wall }}$ & $1000 \mathrm{~K}$ \\
\hline
\end{tabular}

Under these conditions, the impinging shock wave is weak enough so that the flow behind it could still be taken as an undissociated ideal gas. Runs were conducted using 98 by 62 and 194 by 124 stretched grids, such as the one shown in Figure 23. Three different impinging shock locations were used to study their effects, and time accurate calculations were performed using the third-order Additive Semi-implicit Runge-Kutta scheme mentioned earlier. The cases were run until a sustained oscillation in the maximum surface pressure was obtained to ensure all transients had died out. This corresponded to a nondecaying oscillation in the residual between iterations.

\section{Comparison with Analysis}

As a check on the computations, numerical results were compared with analytical predictions for flow variables in thermo-chemical equilibrium behind the interacting shocks. Flow conditions near the shock interaction point were solved using an iterative technique. The analytical model was the ideal dissociating gas model of Lighthill ${ }^{[27]}$, in conjunction with the hydrodynamic jump conditions across the shock waves. The procedure was similar to that used by Sanderson ${ }^{[18]}$ in his analysis of experimental data. The flow field was divided into four regions as shown in Figure 24. In the figure, region 1 is the freestream, region 2 is the flow behind the impinging shock, re- gion 3 is the area behind the upper bow shock, and region 4 is the jet region behind the shock transmitted due to the oblique shock impingement. Jump relations were simultaneously solved across the shocks dividing regions 1 and 3 and dividing regions 2 and 4. Pressure and deflection continuity across the slip line dividing regions 3 and 4 was used as a constraint on the solution.

Figure 25 shows a comparison of numerical and analytic solutions for pressure. The upper figure shows a profile along line a-a in Figure 24, while the lower figure shows a profile along line $b-b$. The computed values for pressure match well with the analytical values. The slight discrepancy in the results are expected because the analytic solution assumed thermochemical equilibrium, and uniform flow in regions 3 and 4 with no viscous dissipation at the interface.

\section{Effect of Shock Impingement Location}

Three cases of different shock impingement locations, measured by the angular displacement of the oblique shock/bow shock interaction point from the horizontal as illustrated in Figure 26, were used to study the effect of impingement location on flow stability. Flow conditions in the freestream and behind the impinging shock were the same for all three cases, except for the impingement locations. The angles of the three cases were $\theta_{i m p}=183.1^{\circ}$ (case A), $186.7^{\circ}$ (case B), and $191.3^{\circ}$ (case C).

Instantaneous temperature contours of each case are shown in Figure 27, and corresponding stream line patterns are given in Figure 28. In case $\mathrm{A}$, the supersonic jet curves upward over the body and does not directly impinge on the body. This is referred to as a type IVa interaction in some of the literature [17]. The vortex structure below the jet (stream trace (a) in Figure 28) is a separation region created due to the fact that the stagnation streamline lies below the jet. The separation bubble formed between the stagnation point, and the jet impingement point, two regions of locally high pressure. This bubble was relatively stable, and was not shed into the flow like the vortices generated in the next case. In case B, the jet can be seen to directly impinge on the body. Vortices are formed near the jet-wall juncture due to high velocity gradients (see stream trace (b) in Figure 28) in these regions of severe turning. This is described in detail below in the discussion of the 
unsteady mechanism of the flow. In case $\mathrm{C}$, the flow is nearly a type III interaction. This flow exhibited the least vortical structure of the three (see stream trace (c) in Fig. 28) and the thinnest shear layer.

The peak heating rates along the cylinder surface for each case are compared in Figure 29. This figure shows surface profiles when heat transfer rates were at their highest levels, as computed on the 98 by 64 grid. The highest heating was produced by case C, and the lowest by the case $A$, because of the absence of direct shock impingement on the body for case A. These results are in qualitative agreement with the heating rate results obtained by Brück ${ }^{[19]}$.

Based on numerical results for ideal gas cases, Lind ${ }^{[14]}$ classified type IV interactions into three categories for stability classification: type $\mathrm{IV}^{+}$, type $\mathrm{IV}$, and type $\mathrm{IV}^{-}$, depending on $\theta_{i m p}$. According to his results, type $\mathrm{IV}^{+}$is steady, type $\mathrm{IV}^{-}$is unsteady, and type IV may be unsteady. The current real gas cases correspond to type $\mathrm{IV}$ for case $\mathrm{A}$ and type $\mathrm{IV}^{-}$ for cases $B$ and $C$. All cases in the present work were found to be unsteady to some degree. Case $\mathrm{C}$ was only mildly unsteady, displaying very little fluctuation in peak surface pressure, and could be essentially considered steady. This was in conflict with Lind's results ${ }^{[14]}$. The possible cause of the discrepancy is that the classifications were based on perfect gas flows, while the work presented here was done using real gas computations. Additionally, the earlier work did not examine as large a $\theta_{i m p}$ as case $\mathrm{C}$, which is very near the type III-IV transition regime. On the other hand, case A showed an intermediate degree of unsteadiness. Case $B$ displayed the highest degree of unsteadiness. Case B is examined in more detail in the next section.

\section{Unsteady Mechanism}

The case of $\theta_{\text {imp }}=186.7^{\circ}$ computed using the 194 by 124 grid exhibited the largest oscillations in peak heating location and magnitude, and jet position, and was therefore the most interesting case, from a time accurate point of view. Instantaneous color contours of this case are given in Figures 34 to 37. In Figure 34, the highest temperatures are near the jet impingement point and behind the strong bow shocks. Behind the bow shocks, the gradual temperature decrease due to thermal relaxation is noticeable. In Figure 35, vortices both above and below the supersonic jet near the jet-wall juncture are evi- dent. These play an important role in the unsteadiness of the jet, as will be discussed later. In Figure 36 , the relaxation of the vibrational energy mode is evident. The distinct differences between the values and shapes of the vibrational temperature contours and the translational temperature contours in Figure 34 demonstrates the degree of thermal nonequilibrium present in the flow. In Figure 37, the supersonic jet is essentially frozen. Meanwhile, just behind the bow shocks, the flow is frozen, but eventually dissociates as it relaxes towards equilibrium. The highest concentrations of $N$ were found near the jet impingement region.

A segment of the time history of the maximum surface pressure is shown in Figure 30. The time axis shows a relative time in seconds and the frame label on the curve correspond to the frame references used throughout this section. Surface heat flux profiles for the frame references are given in Figure 31. The large variation in peak heating rate magnitude can be seen by comparing frames 5 and 21 . Figure 32 shows the maximum enhancement (the ratio of the surface heat flux normalized by the stagnation point heating rate for an undisturbed flow) obtained for this case.

Instantaneous contours taken at these point for translational temperature are shown in Figures 38 to 45 . Corresponding stream line traces are in Figures 46 to 53 , and corresponding $N$ mass fraction contours are shown in Figures 54 to 61 . The oscillation of the supersonic jet can be seen by tracing these contours along the time history. The jet is initially curved upwards, almost in a type IVa configurations as can be seen in Figures 38, 46, and 54 . The jet moves downwards in Figures 39, 47, and 55 until it is aligned nearly normal to the body surface in frame 21 (Figures 40, 48, and 56). At this point, the peak surface pressure and heating rates are at a maximum. This point is also important as it marks a change in direction of the jet motion. After this, the jet moves upwards again until a pressure and heating minimum is reached (frame 45 is close to this minimum point). The mechanism behind this oscillation is related to the relative orientation of the jet to the body, and the shear layers produced along the surface.

Lind $^{[14]}$ found that ideal gas vortices were shed from the jet-body juncture during the course of the jet oscillations and convected off with flow, eventually dissipating in the shear layer. He proposed the vortex shedding as the cause for the unsteadiness 
in the flow field. The real gas flow fields presented here also show a time dependent shedding of vortices. However, unlike Lind's case, the present case shows vortices being shed from both the upper and lower surfaces of the jet. In frames 5 and 13 (Figures $38,39,46,47)$, a vortex can be seen below the jet at the jet-body interface. During this span, the jet is moving downwards, affected by the lower presure of the vortex. A vortex above the jet forms in frame 21 (Figures 40 and 48 ), which the point where the jet nearly normal to the body surface and the surface pressure and heating rates are at a maximum. The upper vortex grows in frames 26 and 35 (Figures 41, $42,49,50)$ as the lower vortex is shed and the jet moves upwards, pulled by the lower pressure of the upper vortex, which is itself shed into the flow. As the upper vortex progresses along the upper shear layer, its lower pressure creates and expansion region in the layer, as can be seen by the growth of the layer in Figures 50 to 52 . In order to turn the flow back in line with the body surface, a shock is formed within the shear layer and progresses through the layer just ahead of the vortex. In Figure 43, this shock forms near the body at the $y=0$ point. In Figures 44 and 45 , the shock travels with the flow ahead of the vortex (compare the location of the wave with the location of the vortex in frames 40,45, and 50). During this span, the lower vortex weakens and eventually dissipates as shown in Figures 51 to 53.

This vortex shedding is consistent with prior findings for ideal gases ${ }^{[14]}$. However, unlike the ideal gas case, the present case shows vortices being shed from both the upper and lower surfaces of the jet. The two vortices are shed out of phase with each other, as can be seen in Figures 50 to 53. This out of phase vortex shedding, shown schematically in Figure 33, was the reason why this case (case $B$ ) was the most unsteady of the three cases examined. In case A, the separation vortex below the jet appeared bound, and was not shed off into the flow. As a result, it contributed relatively little to the jet unsteadiness relative to an unsteady shed vortex. In case $\mathrm{C}$ the jet flow along the lower surface was not turned as severely as in the other two cases (compare the stream lines in Fig. 28) and a vortex is not formed below the jet.

\section{$\underline{\text { Real Gas Effects }}$}

The nonequilibrium nature of the computed flow fields can be seen in the instantaneous dissociated nitrogen mass fraction contours in Figures 54 to 61 .
As shown in these contours, there is significant variation in $N$ composition throughout the flow field at various times. The maximum $N$ mass fraction observed was 0.12 just behind the terminating jet shock in frame 21. The region showing the next highest degree of dissociation was the area behind the upper bow shock where it was almost normal to the free stream. $N$ mass fractions in this region reached as high as 0.10 . The jet, on the other hand, was essentially frozen because the transmitted oblique shock was considerably weaker than the bow shocks. An interesting feature of the flow occurs along the wall. In Figure 55, a disturbance of high $N$ concentration can be seen forming just behind the terminating jet shock. This disturbance travels along the upper surface in Figures 56 through 61. Behind this, a region of low $N$ concentration is shed in to shear layer when the jet begins to turn upwards in Figure 58. These concentration disturbances are then convected off through the shear layer.

The structure of the flow field changes drastically in the presence of real gas effects. For example, there is a dramatic decrease in shock stand off distance in the real gas case compared to an ideal gas case, and thus large changes in jet length. The effects that this has in the present case can be seen by comparing the case $\mathrm{B}\left(\theta_{i m p}=186.7^{\circ}\right)$ with the ideal gas case studied by Zhong ${ }^{[11]}\left(\theta_{i m p}=188.5^{\circ}\right)$. The levels of both heat transfer and peak surface pressure enhancement were lower for the real gas computations. Case B produced a heating enhancement ratio (the ratio of peak heating to the peak heating rate without the impinging oblique shock) of 17.2 , as illustrated in Figure 32, and a peak pressure enhancement ratio of 9.6. The ideal gas case resulted in ratios of 20 and 12.1, respectively. This reduction in the heat transfer and pressure load enhancement due to real gas effects is qualitatively similar to Sanderson's analysis of stagnation point heat transfer rates using the Fay-Riddel model ${ }^{[18]}$ for a nonequilibrium flow, but disagrees with the same sort of analysis for equilibrium flow results. Sanderson's assumption for the nonequilibrium flow solutions was that the reaction rates in the jet region were relatively small, which is consistent with the computational result of a frozen jet. Therefore, the current numerical results would correspond to Sanderson's nonequilibrium solutions. 


\section{Conclusions}

Steady shock/boundary layer and unsteady type IV shock interference interactions were studied using numerical simulations. The shock/ boundary layer case was computed for three different models, ideal gas, vibrationally excited but chemically frozen, and full thermo-chemical nonequilibrium, and four different freestream temperatures to study the effects of thermo-chemical nonequilibrium. The unsteady type IV case was run for three different impinging shock locations to study the effect of this on the stability of the flow. Additionally, the most unsteady case was closely examined to study the real gas effects on the unsteady mechanism of the flow.

In the shock/boundary layer interaction, the effect of thermo-chemical nonequilibrium was found to reduce the surface heating rates, and the size of the shock induced separation region. For the real gas model, raising the freestream temperature also showed the same trends up to a point. For sufficiently high freestream enthalpy, temperatures at the shock impingement point near the back of the separation bubble were high enough to cause significant nitrogen dissociation in addition to the oxygen dissociation present in the cooler flows. This additional dissociation sustained the adverse pressure gradient behind the impinging shock further than in the lower enthalpy flows, thus causing the separation region to grow in size relative to the cooler flow cases.

For the type IV shock interference heating problem, the location of the impinging shock was found to affect the stability of the flow. The flow near the type III-IV transition region was the most stable; flow where the supersonic jet can coincide with a stagnation streamline was the most unsteady. The unsteadiness was found to be related to the development and shedding of vortex structures near the jet-wall interface. The largest degree of unsteadiness was the result of alternating shed vortices both above and below the jet. The effect of thermochemical nonequilibrium was observed to be a decrease in peak heating and peak pressure enhancement relative to ideal gas results, which are consistent with available analytical results.

\section{Further Studies}

A parametric study on the effects of impinging shock location and degree of nonequilibrium will be carried out to further understand the influence real gas effects have on these flows. The popular Park chemistry model for air ${ }^{[23]}$ will be added. Additionally, an analytical study of the unsteady processes associated with the jet along with a Fourier analysis of of the unsteadiness are planned, and simulations for comparison with Sanderon's shock tube data ${ }^{[18]}$ will be run. A turbulence model may be added if it is deemed necessary.

\section{Acknowledgements}

This research was supported by the Air Force Office of Scientific Research under grant numbers F49620-94-1-0019 and F49620-95-1-0405 monitored by Dr. Len Sakell. Cray CPU time was provided by the CEWES High Performance Computing Center. The authors would like to thank Dr. Stefan Brück of the DLR Institute of Fluid Mechanics for a preprint of his paper.

More information on this work and related ongoing research can be found on the WWW at http://cfdlab5.seas.ucla.edu

\section{References}

[1] J. Delery. Shock/shock and shock wave/boudnary layer interactions in hypersonic flows. In Aerothermodynamics of $\mathrm{Hy}$ personic Vehicles. AGARD-R-761, 1989.

[2] G. Ben-Dor. Shock Wave Reflection Phenomena. Springer-Verlag, 1992.

[3] I. I. Glass. Some aspects of shock-wave research. AIAA Journal, 25:214-229, 1987.

[4] C. A. Ballaro and J. D. Anderson Jr. Shock Strength Effects on Spearated Flows in NonEquilibrium Chemically Reacting Air Shock Wave/Boundary Layer Interaction. AIAA Paper 91-0250, 1991.

[5] A. A. Grumet, J. D. Anderson Jr., and M. J. Lewis. A Numerical Study of Shock Wave/Boundary Layer Interaction in Nonequilibrium Chemically Reacting Air: The Effects of Catalytic Walls. AIAA Paper 91-0245, 1991. 
[6] R. L. Deshambault and I. I. Glass. An update on non-stationary oblique shock-wave reflections: Actual isopycnicsand numerical experiments. Journal of Fluid Mechanics, 131:27$57,1983$.

[7] H. M. Glaz, P. Collela, I. I. Glass, and R. L. Deschambault. A numerical study of oblique shock-wave reflections with experimental comparisons. Proceedings of the Royal Society of London A, 398:117-140, 1983.

[8] B. Edney. Anomalous Heat Transfer and Pressure Distributions on Blunt Bodies at Hypersonic Speeds in the Presence of an Impinging Shock. Aeronautical Research Inst. of Sweden, FFA Rept. 115, Feb. 1968.

[9] A. R. Wieting and M. S. Holden. Experimental shock wave interference heating on a cylinder at mach 6 and 8. AIAA Journal, 27:1557-1565, 1989.

[10] M. S. Holden. Shock-Shock Boundary Layer Interaction. AGARD Rept. No. 764, July 1989.

[11] X. Zhong. Application of essentially nonoscillatory schemes to unsteady hypersonic shockshock interference heating problems. AIAA Journal, 32:1606-1616, 1994.

[12] G. H. Klopher and H. C. Yee. Viscous Hypersonic Shock-on-shock Interaction on Blunt Cowl Lips. AIAA Paper 88-0233, 1988.

[13] G. Gaitonde and J. S. Shang. The Performance of Flux-Split Algorithms in High-Speed Viscous Flows. AIAA Paper 92-0186, 1992.

[14] C. A. Lind and M. J. Lewis. A Numerical Study of the Unsteady Processes Associated with the Type IV Shock Interaction. AIAA Paper 93$2479,1993$.

[15] C. Chiu and X. Zhong. Simulation of Transient Hypersonic Flow Using the ENO Schemes. AIAA Paper 95-0469, 1995.

[16] R. K. Prabhu, J. R. Stewart, and R. R. Thareja. Shock Interference Studies on a Circular Cylinder at Mach 16. AIAA Paper 90-0606, 1990.

[17] J. W. Keyes and F. D. Hains. Analytical and Experimental Studies of Shock Interference Heating in Hypersonic Flows. NASA TN D$7139,1973$.
[18] S. R. Sanderson. Shock Wave Interaction in Hypervelocity Flow. $\mathrm{PhD}$ thesis, California Institute of Technology, 1995.

[19] S. Brück. Investigation of Shock-Shock Interactions in Hypersonic Reentry Flows. Presented at the 20th International Symposium on Shock Waves, 1995.

[20] J. D. Anderson jr. Hypersonic and High Temperature Gas Dynamics. McGraw-Hill, Inc., 1989.

[21] G. V. Candler. The Computation of Weakly Ionized Hypersonic Flows in Thermo-Chemical Nonequilibrium. PhD thesis, Stanford University, 1988.

[22] T. Gökçen. The Computation of Hypersonic Low Density Flows with Thermochemical Nonequilibrium. PhD thesis, Stanford University, 1989.

[23] C. Park. Nonequilibrium Hypersonic Aerothermodynamics. Wiley Interscience, 1990.

[24] J. Häuser, J. Muylaret, H. Wong, and W. Berry. Computational aerothermidynamics for $2 \mathrm{~d}$ and 3d space vehicles. In T. K. S. Murthy, editor, Computational Methods in Hypersonic Aerodynamics. Kluwer Academic Publishers, 1991.

[25] B. Grossman and P. Cinella. Flux split algorithms with non-equilibrium chemistry and vibrational relaxation. Journal of Computational Physics, 88:131-168, 1990.

[26] X. Zhong. New High-Order Semi-Implicit Runge-Kutta Schemes for Computing Transient Nonequilibrium Hypersonic Flow. AIAA Paper 95-2007, 1995.

[27] W. G. Vincenti and C. H. Kruger. Introduction to Physical Gas Dynamics. John Wiley and Sons, Inc., 1965.

[28] J. N. Moss, G. A. Bird, and V. K. Dorga. Nonequilibrium Thermal Radiation for an Aerospace Flight Experiment Vehicle. AIAA Paper 88-0081, 1988.

[29] C. R. Wilke. A viscosity equation for gas mixtures. Journal of Chemical Physics, 18(4):517$519,1950$.

[30] R. C. Millikan and D. R. White. Systematics of vibrational relaxation. Journal of Chemical Physics, 12:3209-3213, 1963. 
[31] C. Park. Assesment of two-temperature kinetic model for ionizing air. Journal of Thermophysics and Heat Transfer, 3:233-244, 1989.

[32] L. C. Hartung, R. A. Mitcheltree, and P. A. Gnoffo. Coupled radiation effects in thermochemical nonequilibrium shock-capturing flowfield calculations. Journal of Thermophysics and Heat Transfer, 8:244-250, 1994.

[33] J. G. Parker. Rotational and vibrational relaxation in diatomic gases. Physics of Fluids, 2(4):449-462, 1959.

[34] P. L. Roe. Characteristic based schemes for the euler equations. Annual Review of Fluid $\mathrm{Me}$ chanics, 18:337-365, 1986.

[35] B. Grossman and P. Cinella. Flux split algorithms for hypersonic flows. In T. K. S. Murthy, editor, Computational Methods in Hypersonic Aerodynamics. Kluwer Academic Publishers, 1991.

[36] D. Gaitonde and J. S. Shang. Accuracy of fluxsplit algorithms in high-speed viscous flows. AIAA Journal, 31:1215-1221, 1993.

[37] R. J. Leveque. Numerical Methods for Conservation Laws. Birkhäuser, 1992.

[38] H. M. Glaz, P. Collela, J. P. Collins, and R. E. Ferguson. Nonequilibrium effects in oblique shock-wave reflection. AIAA Journal, 26:698$705,1988$.

[39] H. G. Hornung. Non-equilibrium dissociating nitrogen flow over spheres and circular cylinders. Journal of Fluid Mechanics, 53:149-176, 1972.

[40] G. V. Candler. On the Computation of Shock Shapes in Nonequilibrium Hypersonic Flows. AIAA Paper 89-0312, 1989. 


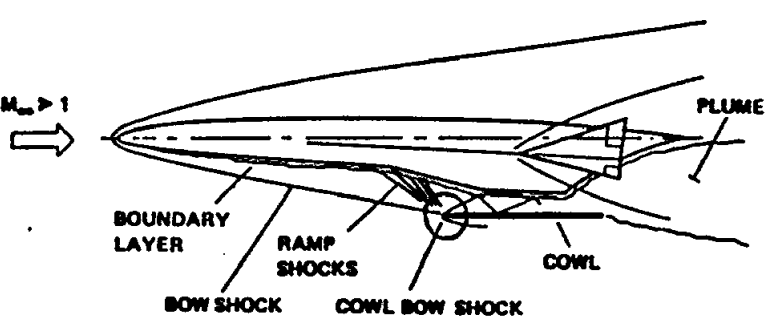

Figure 1: Typical Hypersonic Vehicle and Associated Shock Interaction

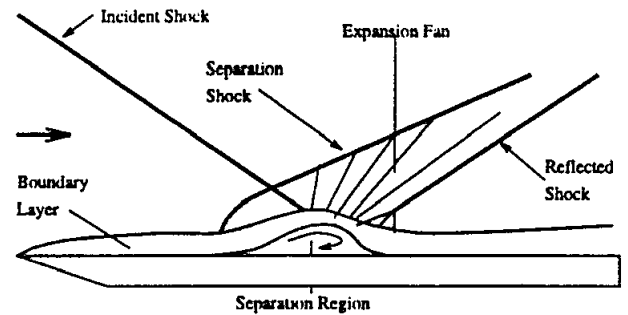

Figure 2: Schematic for Steady Shock-Boundary Layer Interaction

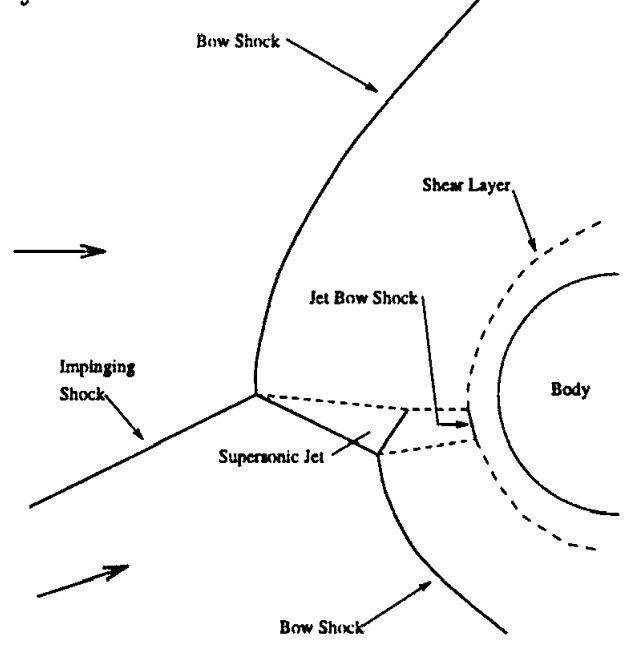

Figure 3: Schematic of Shock on Shock Interference Heating

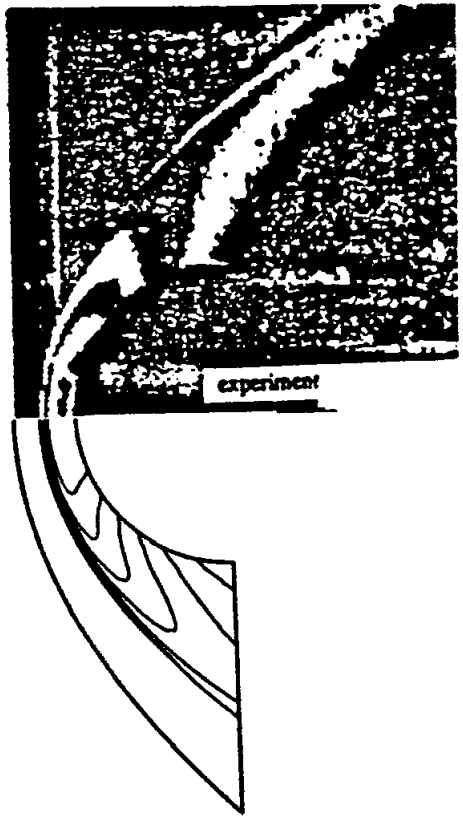

Figure 4: Computed density contours compared with experimental results from Hornung.

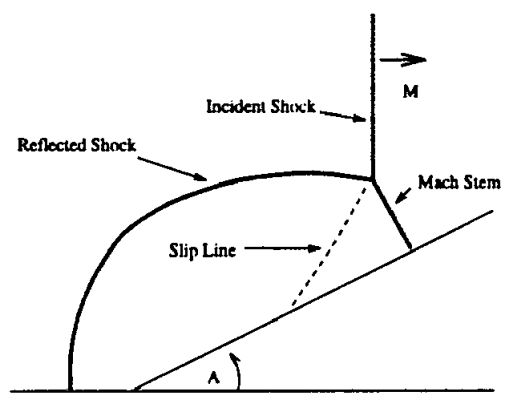

Figure 5: Schematic for Pseudostationary Mach Reflection 


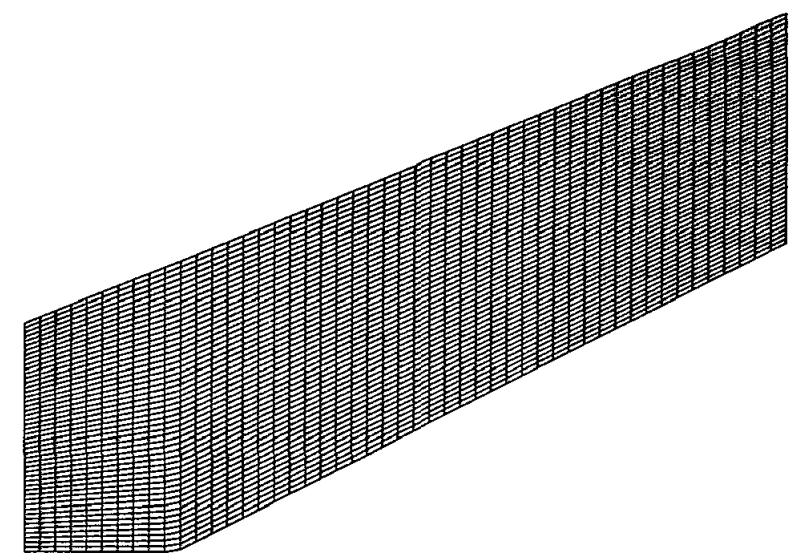

Figure 6: Computational grid for the pseudo steady Mach reflection case. Grid shown is coarser than actual for clarity.

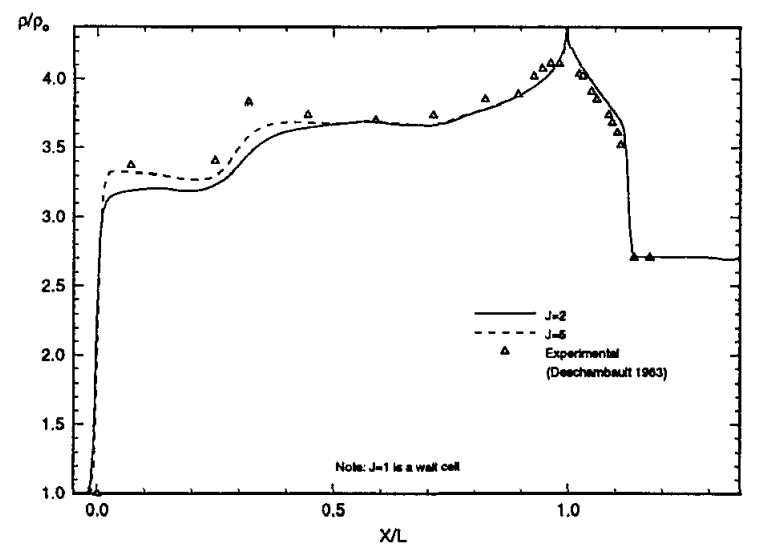

Figure 7: Density profiles $\left(\rho / \rho_{0}\right)$ for computational cells $j=2$ and $j=4$ compared with experimental data (inviscid ideal gas).

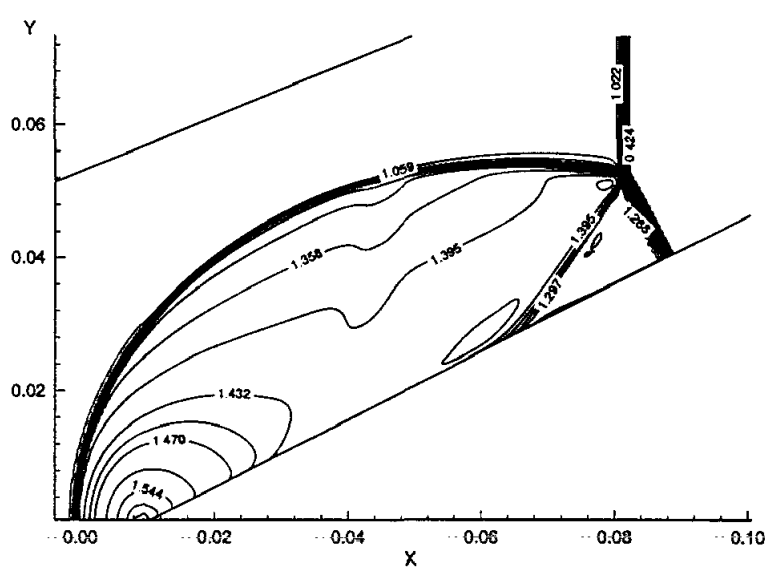

Figure 8: Mach reflection density contours $\left(\mathrm{kg} / \mathrm{m}^{3}\right)$ for inviscid ideal gas case.

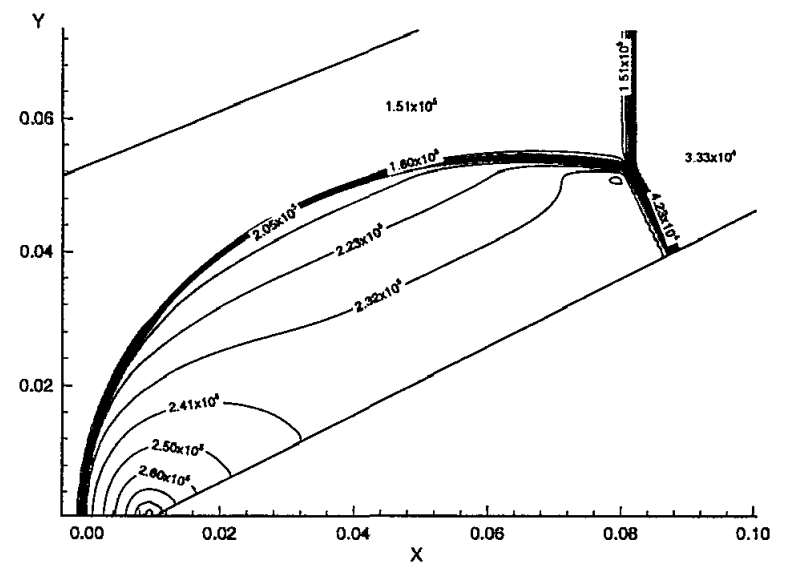

Figure 9: Mach reflection pressure contours $\left(N / m^{2}\right)$ for inviscid ideal gas case.

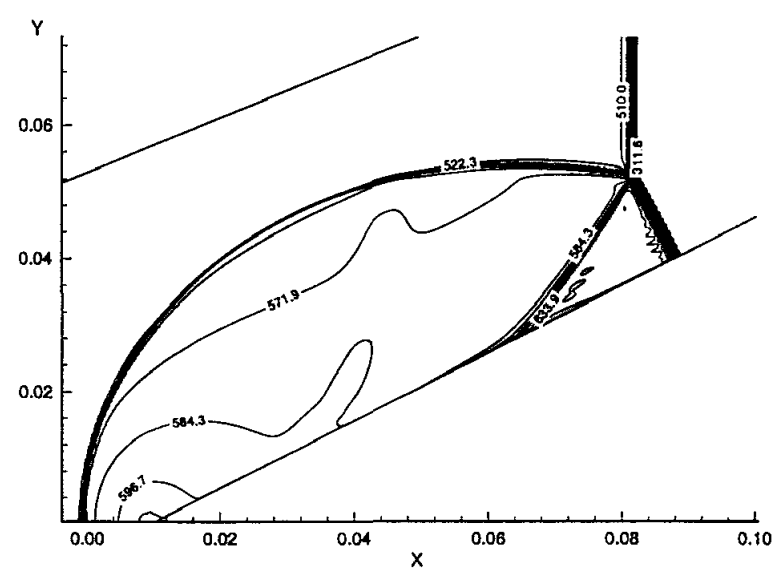

Figure 10: Mach reflection temperature contours $(K)$ for inviscid ideal gas case. 


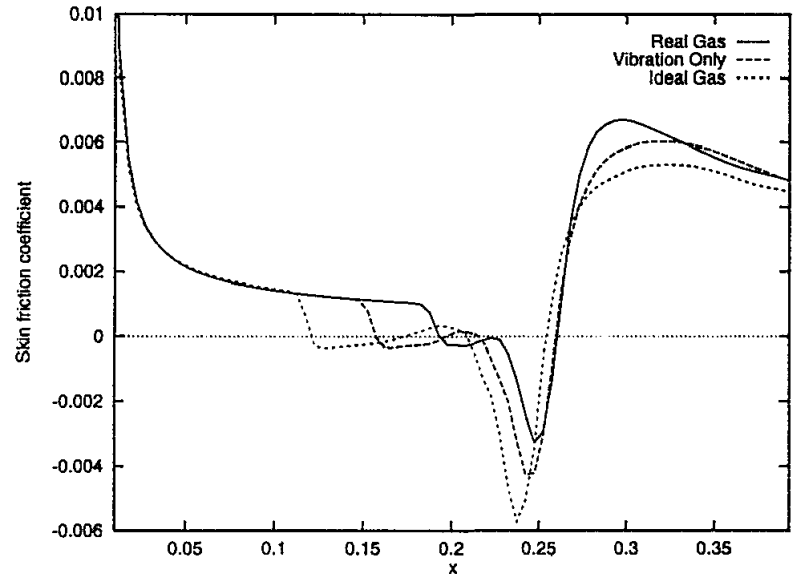

Figure 11: Flat plate $c_{f}$ distribution for different models

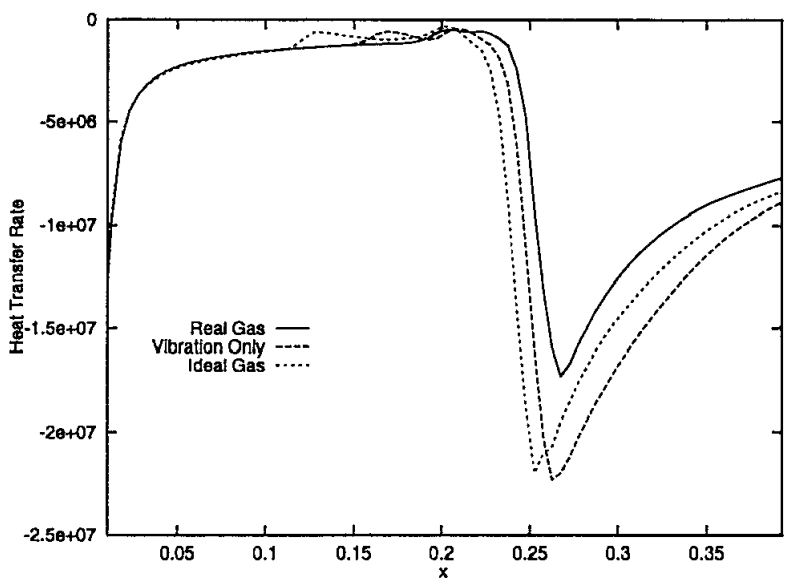

Figure 13: Flat plate $\dot{Q}\left(J / m^{2} s\right)$ for different models

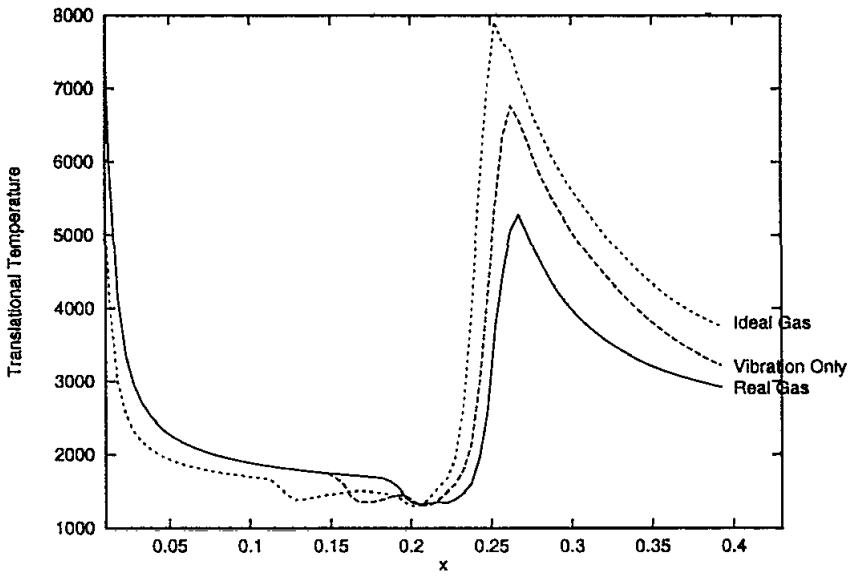

Figure 14: Translational temperature (K) along the plate for different models
Figure 12: Flat plate $p / p_{\infty}$ distribution for different models

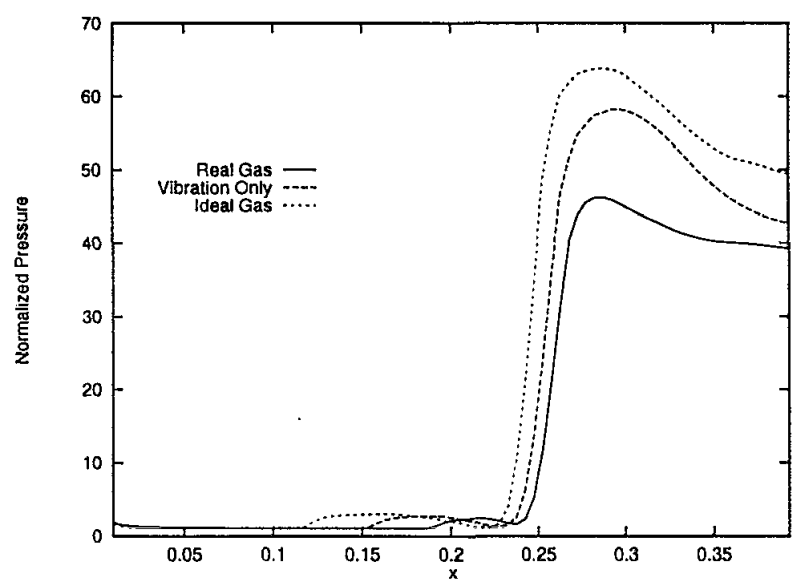




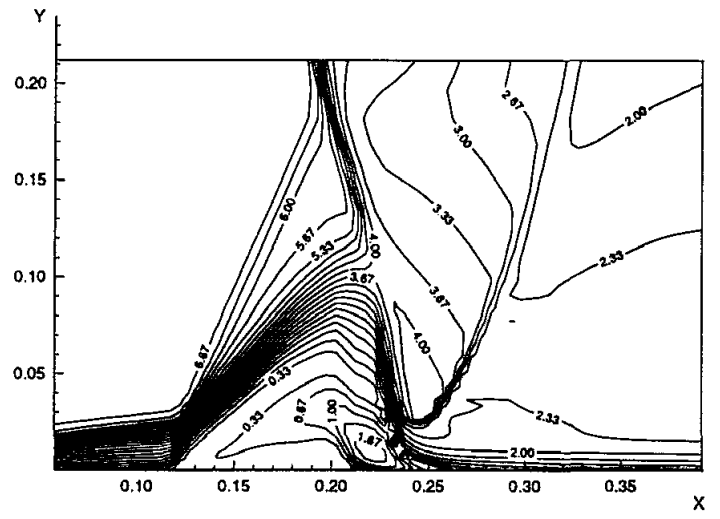

(a)

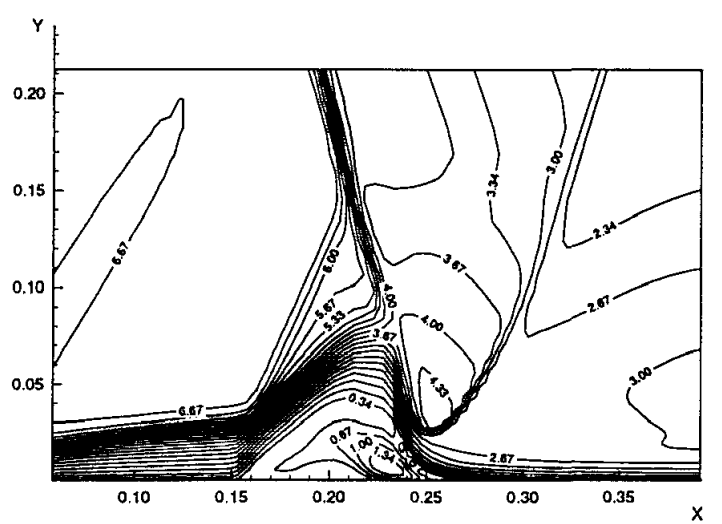

(b)

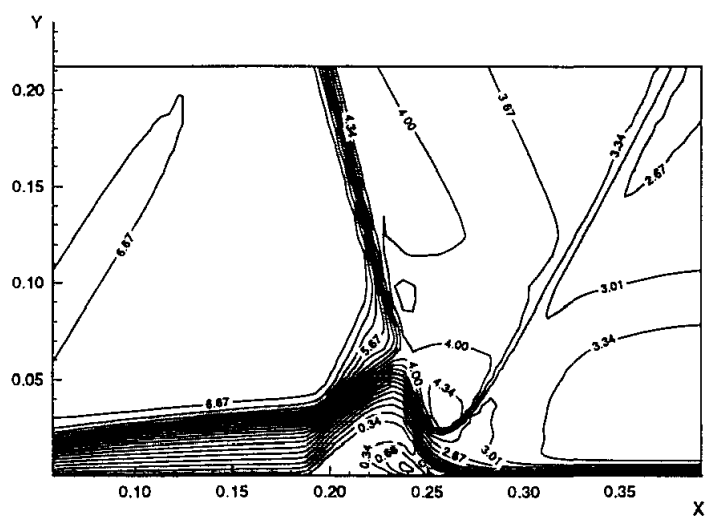

(c)

Figure 15: Mach number contours for Mach 7 shock/boundary layer interaction with different models: (a) Ideal gas, (b) Vibrational excitation and frozen chemistry,(c) Full nonequilibrium gas. (All: $\left.T_{\infty}=1600^{\circ} \mathrm{K}\right)$

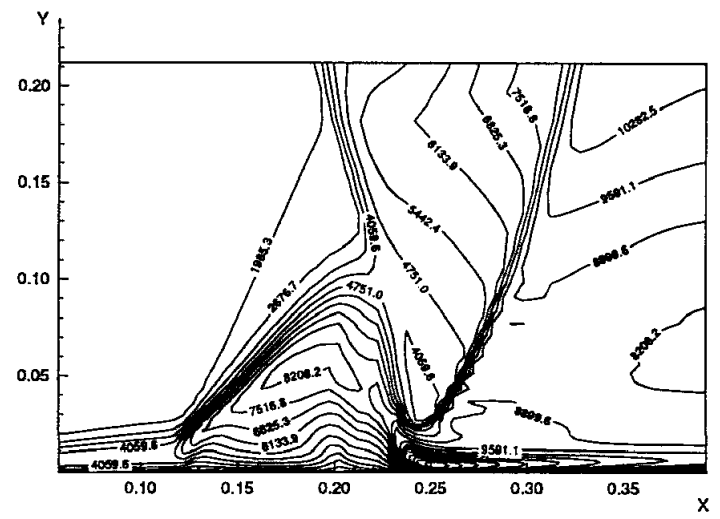

(a)

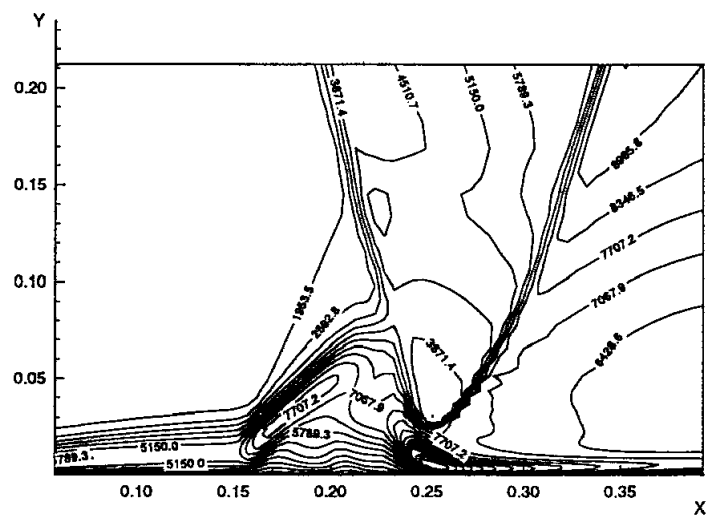

(b)

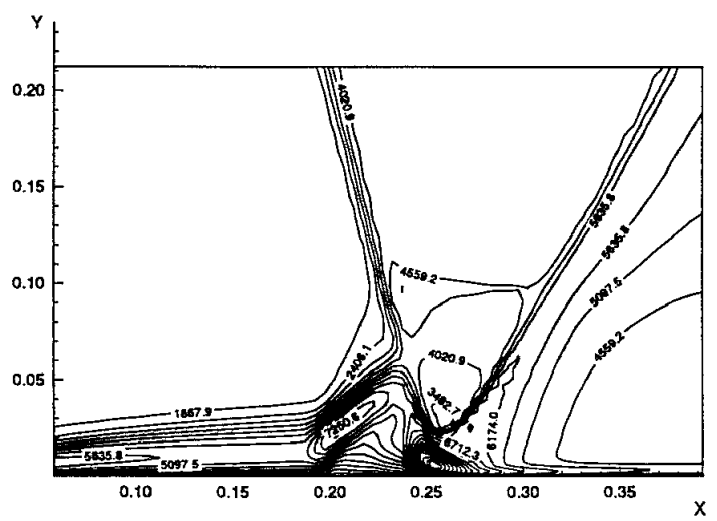

(c)

Figure 16: Translational temperature $(K)$ contours for Mach 7 shock/boundary layer interaction with different models: (a) Ideal gas, (b) Vibrational excitation and frozen chemistry, (c) Full nonequilibrium gas.(All: $T_{\infty}=1600^{\circ} \mathrm{K}$ ) 


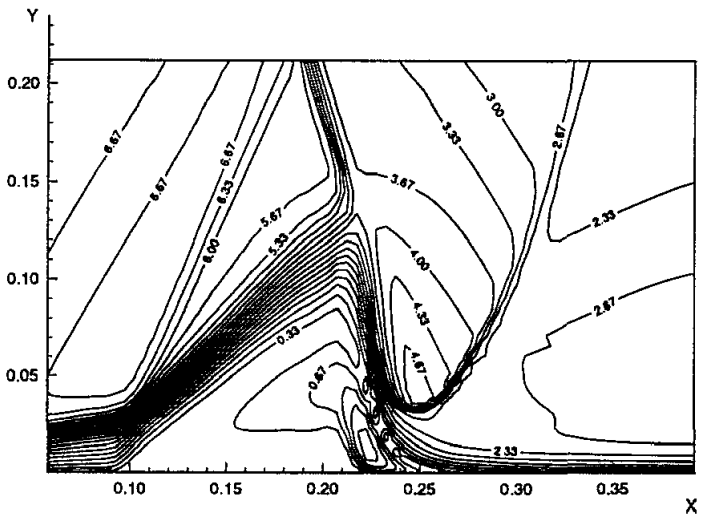

(a)

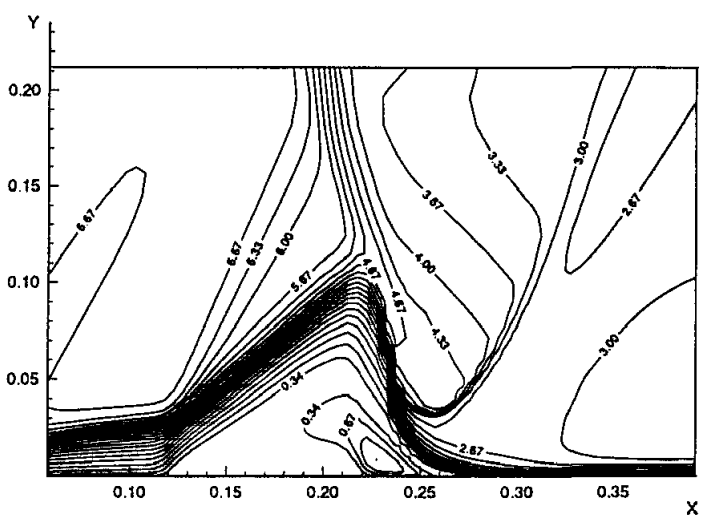

(b)

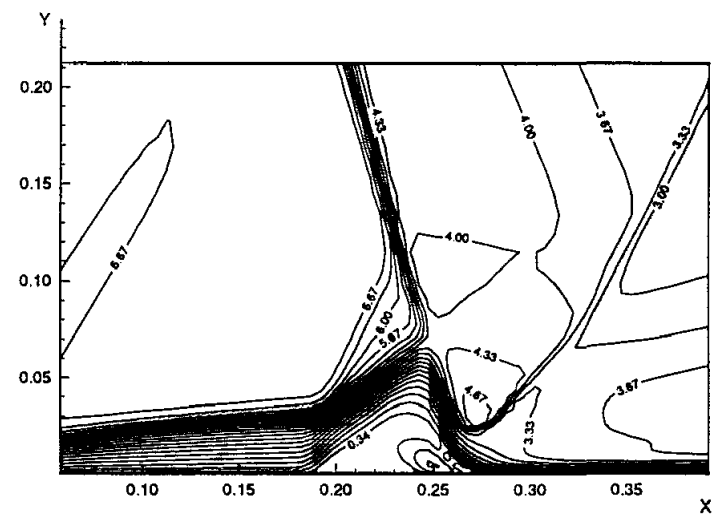

(c)

Figure 17: Mach number contour for Mach 7 shock/boundary layer interaction with full nonequilibrium and different enthalpies: (a) $T_{\infty}=700^{\circ} \mathrm{K}$, (b) $T_{\infty}=1200^{\circ} \mathrm{K}$, (c) $T_{\infty}=2000^{\circ} \mathrm{K}$.

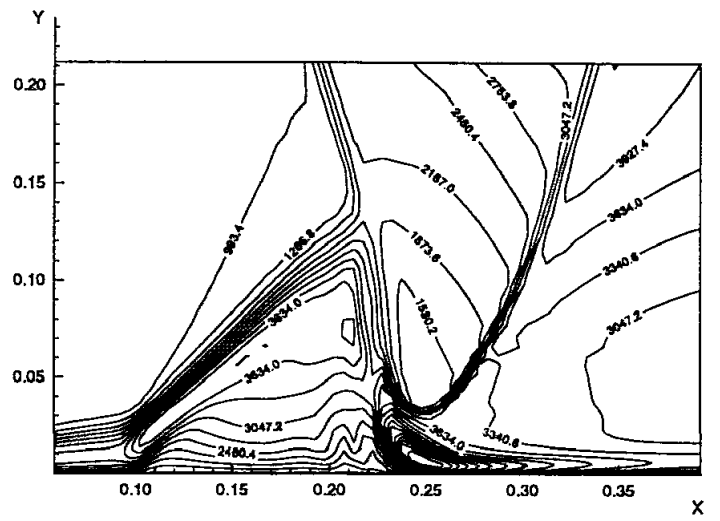

(a)

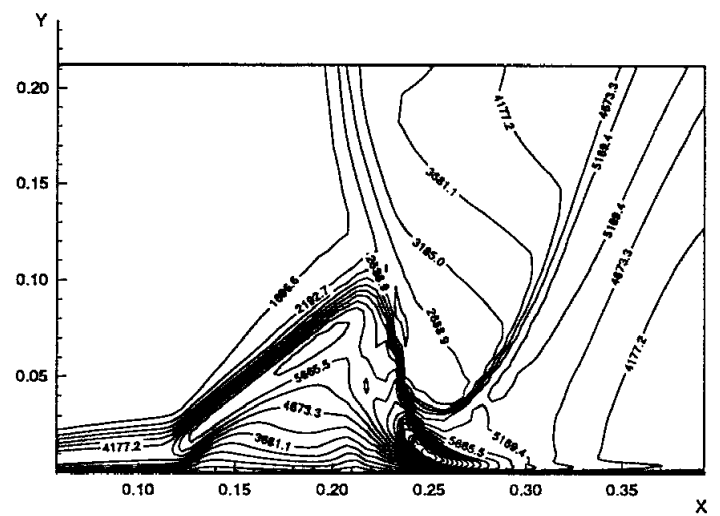

(b)

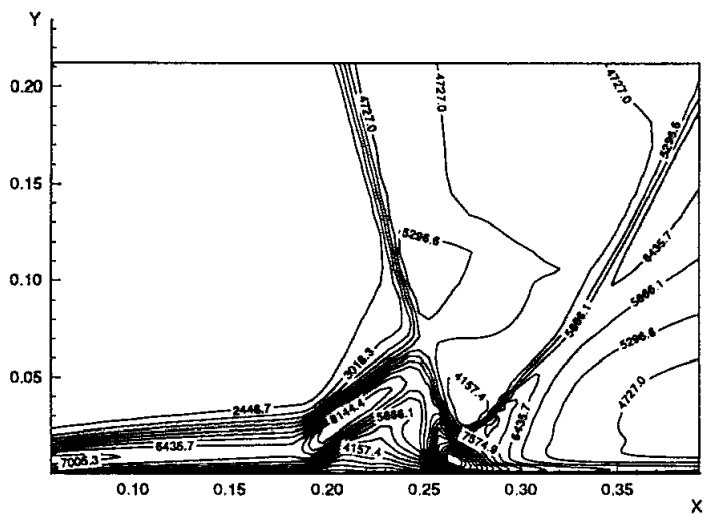

(c)

Figure 18: Translational temperature $(K)$ contour for Mach 7 shock/boundary layer interaction with full nonequilibrium and different enthalpies: (a) $T_{\infty}=700^{\circ} \mathrm{K}$, (b) $T_{\infty}=1200^{\circ} \mathrm{K}$, (c) $T_{\infty}=$ $2000^{\circ} \mathrm{K}$. 


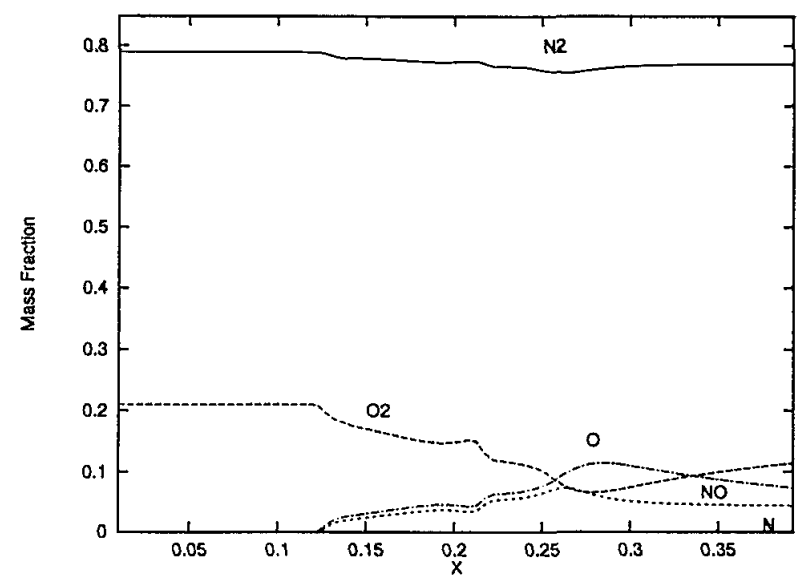

(a)
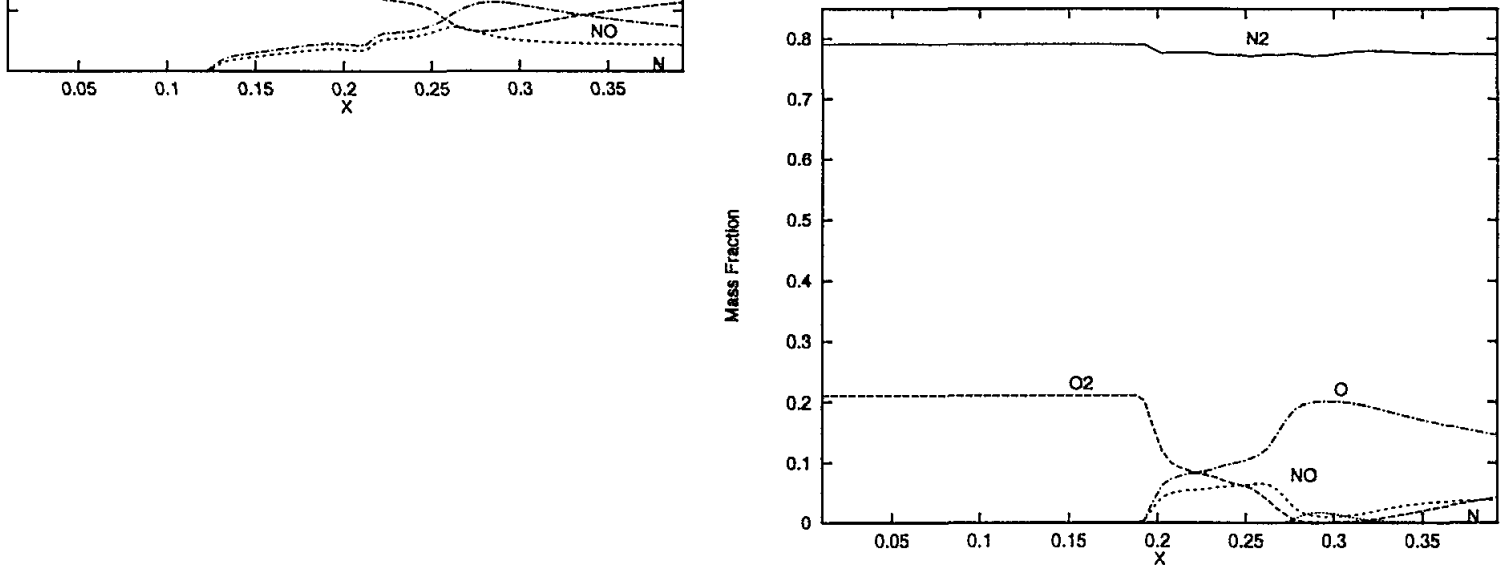

(c)

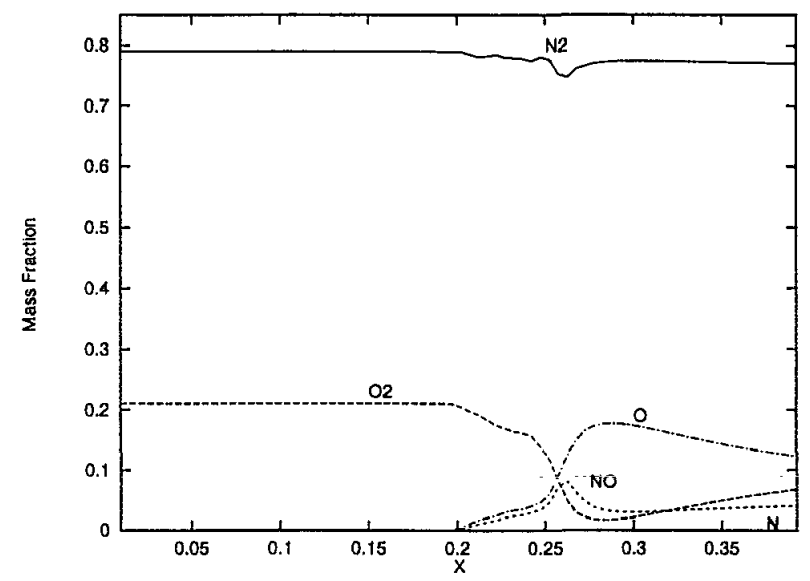

(b)
Figure 19: Mass fractions along the wall for the Mach 7 flat plate with full nonequilibrium and different enthalpies: (a) $T_{\infty}=1200^{\circ} \mathrm{K}$, (b) $T_{\infty}=$ $1600^{\circ} \mathrm{K}$, (c) $T_{\infty}=2000^{\circ} \mathrm{K}$. 


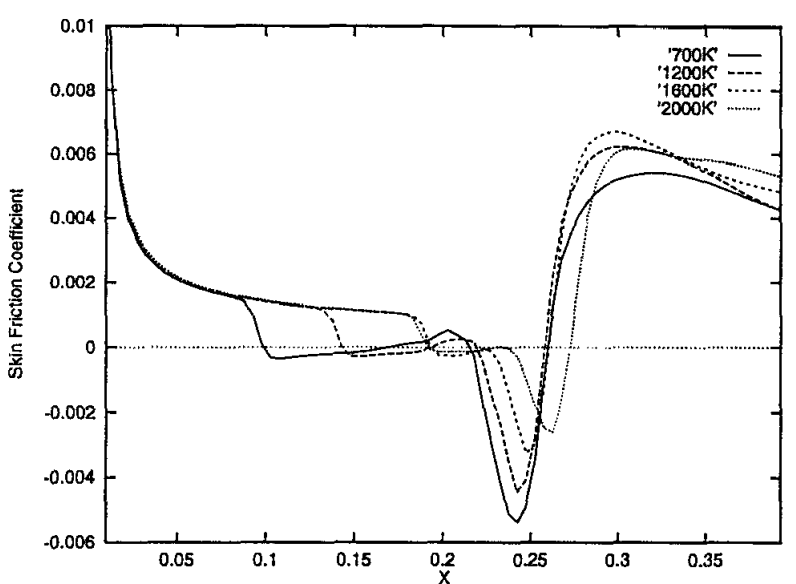

Figure 20: Flat plate $c_{f}$ distribution for different freestream temperatures

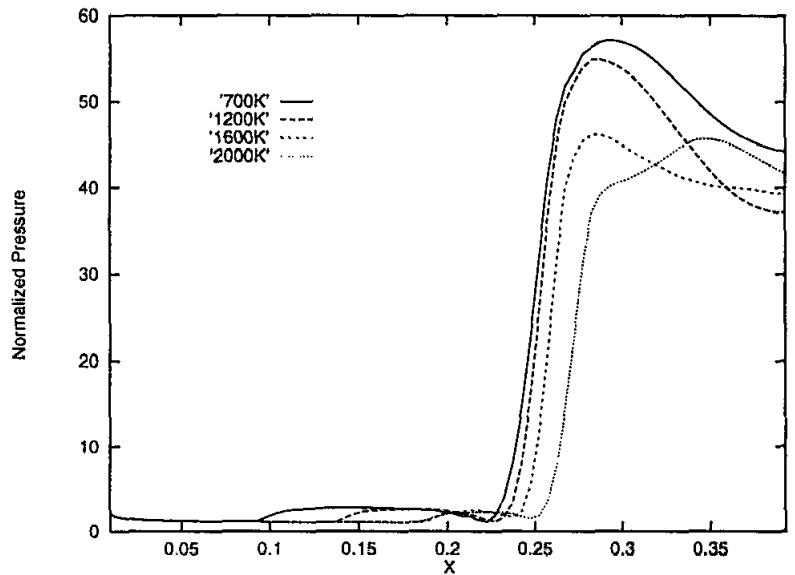

Figure 21: Flat plate $p / p_{\infty}$ distribution for different freestream temperatures

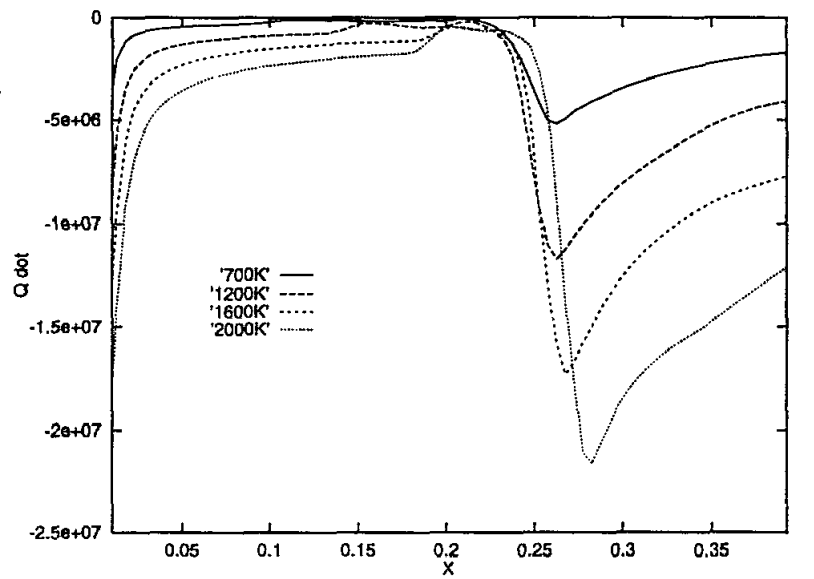

Figure 22: Flat plate $\dot{Q}\left(J / m^{2} s\right)$ distribution for different freestream temperatures 


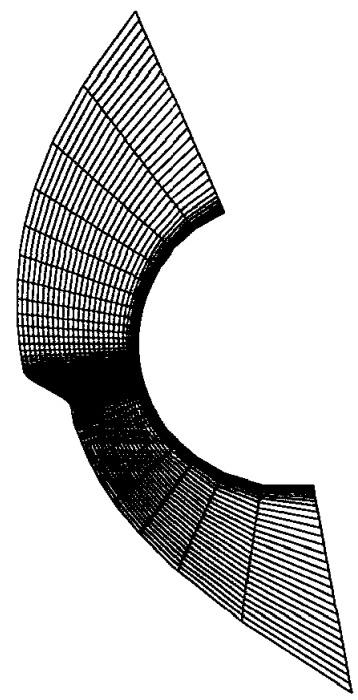

Figure 23: Sample gird used in Type IV shock interference computations. Every third grid line is shown for clarity.

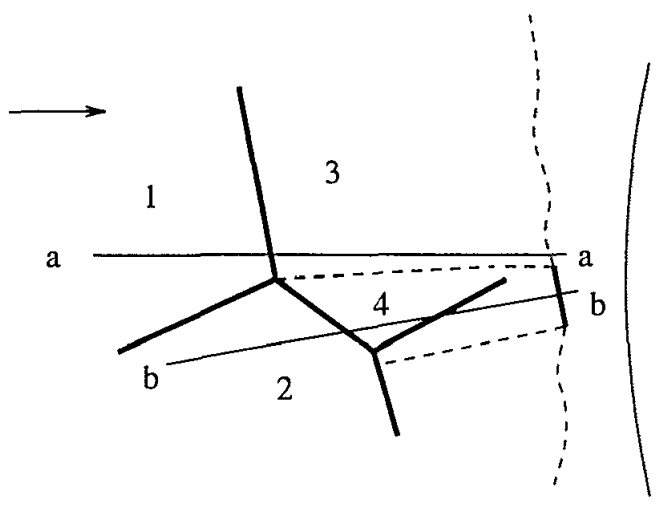

Figure 24: Schematic to illustrate analytic solution. Lines $\mathrm{a}=\mathrm{a}$ and $\mathrm{b}-\mathrm{b}$ indicate lines along which comparison of numerical and analytic solutions were made.
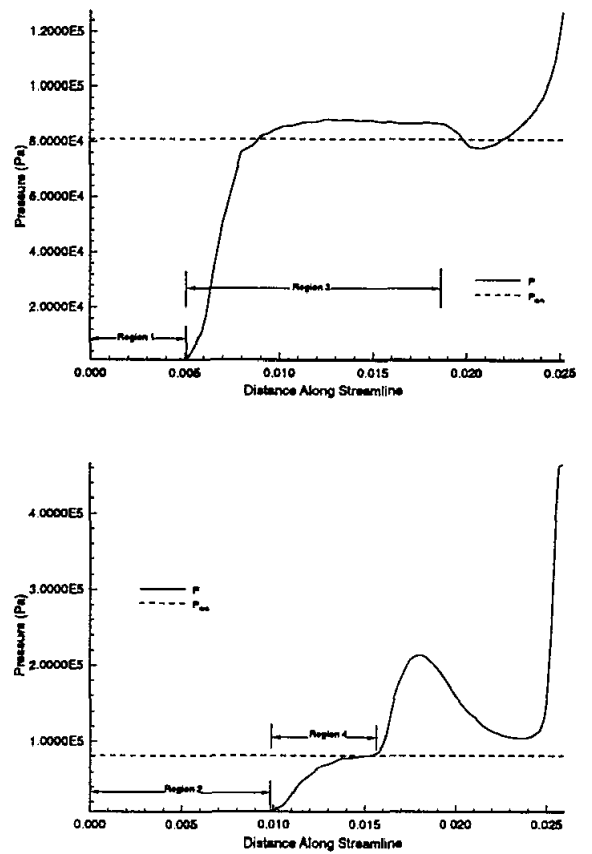

Figure 25: Comparison of numerical and analytical results. Dashed lines indicate analytic solutions in region 3 (upper) and region 4 (lower).

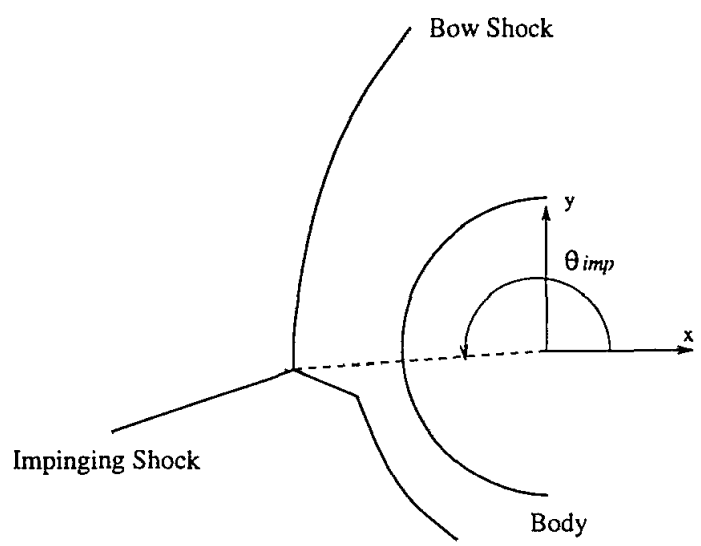

Figure 26: Definition of $\theta_{i m p}$ for shock impingement location study. 

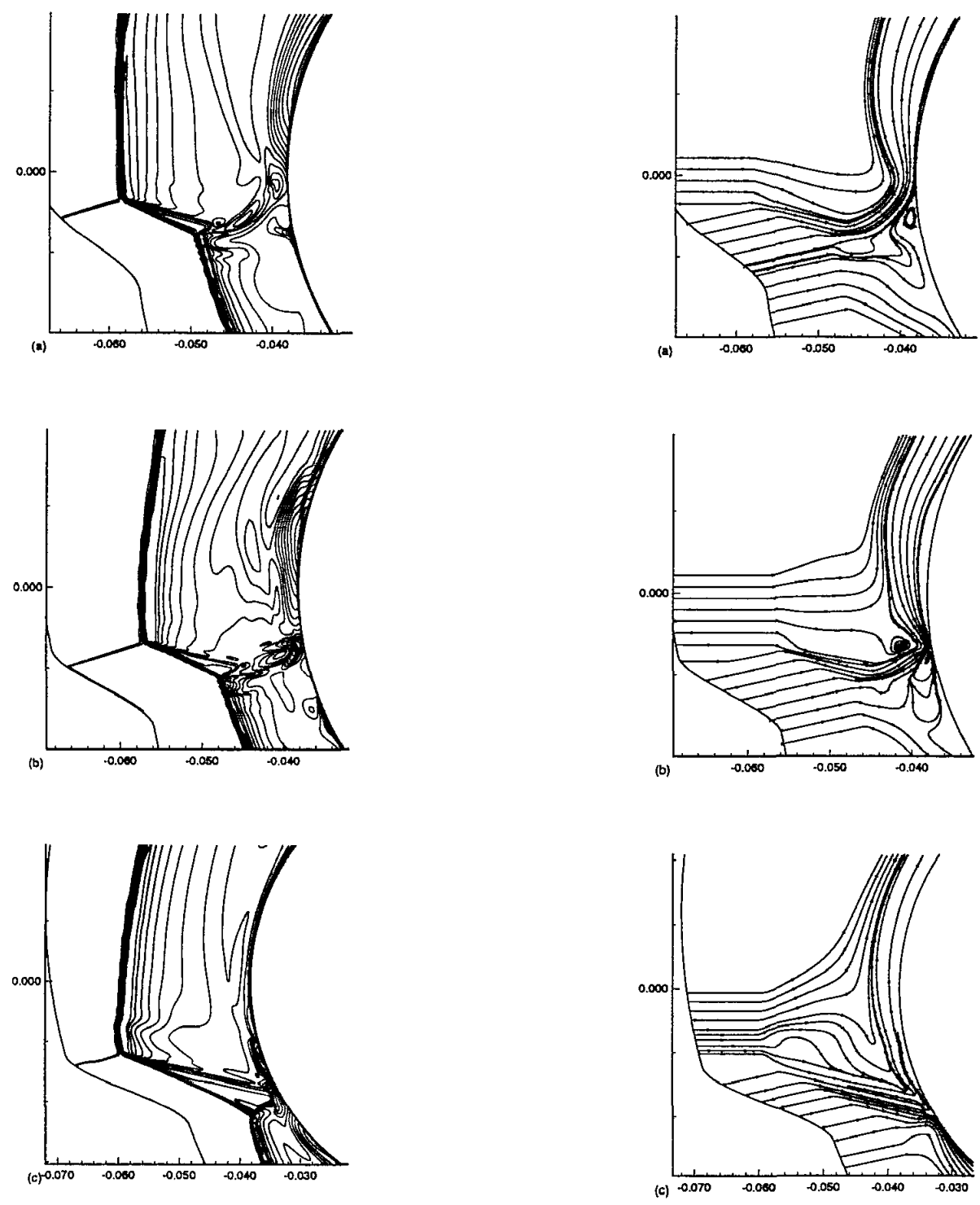

Figure 27: The effect of shock impinging location (contours are translational temperature). (a) $\theta_{i m p}=$ $183.1^{\circ}$ (b) $\theta_{i m p}=186.7^{\circ}$ (c) $\theta_{i m p}=191.3^{\circ}$.
Figure 28: Representative stream traces for the same flows as shown in the previous figure. 


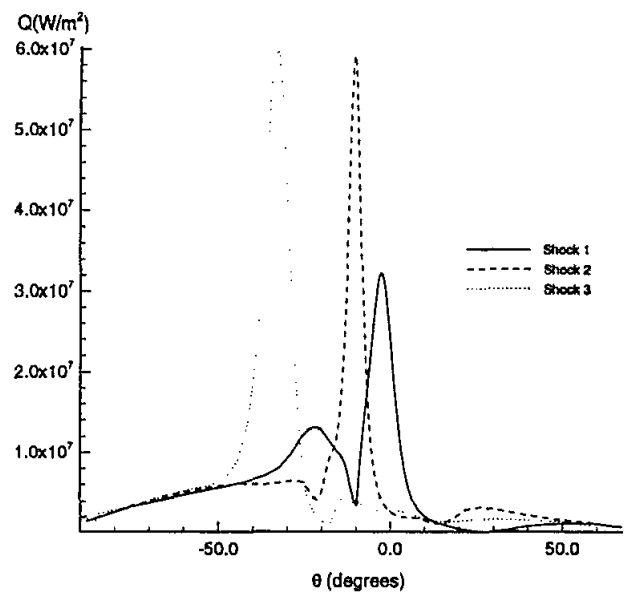

Figure 29: Surface heat transfer profiles for the three different shock locations.

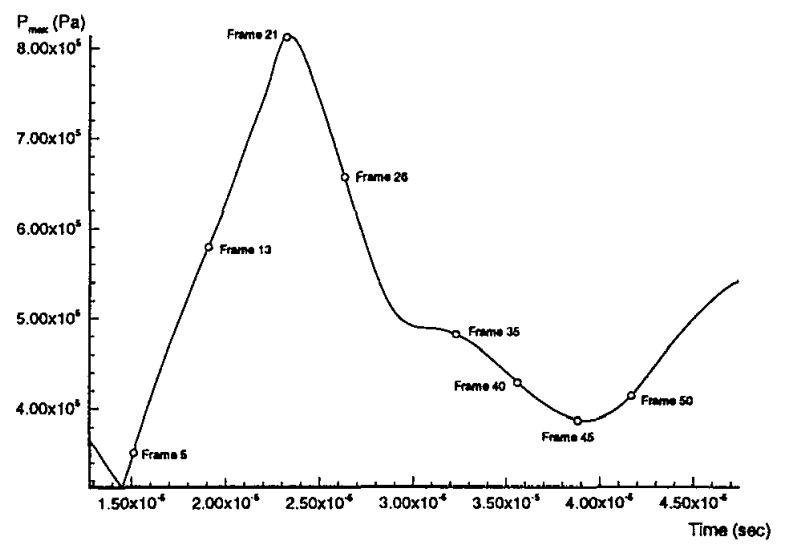

Figure 30: Maximum surface pressure $(\mathrm{Pa})$ time history for case $\mathrm{B}$ type IV interaction on 194 by 124 grid.

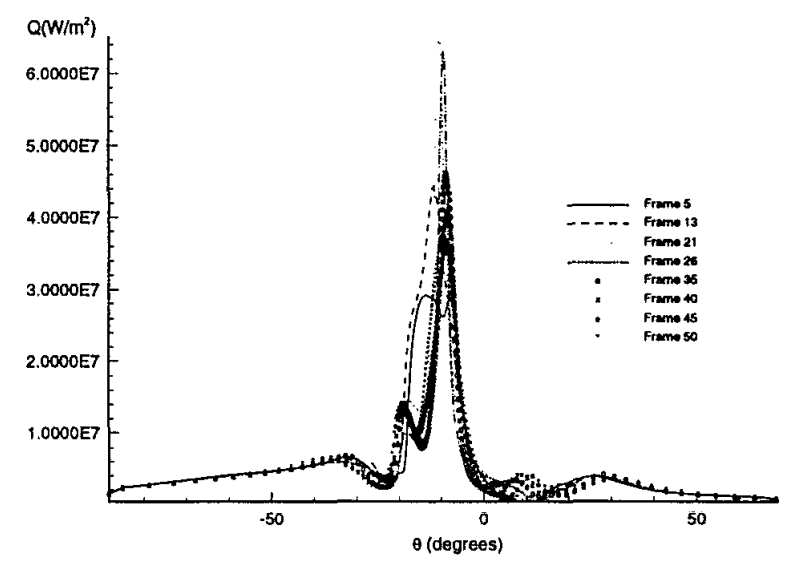

Figure 31: Surface heat transfer profiles at various times for $\theta_{i m p}=186.7^{\circ}$.

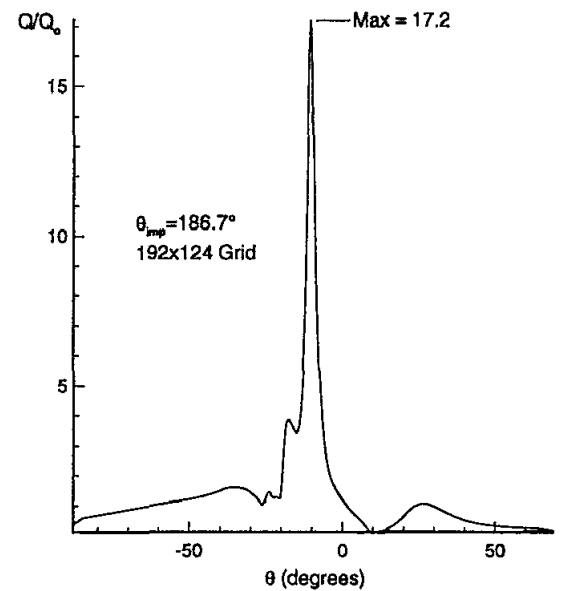

Figure 32: Surface heating rate normalized by undisturbed peak heating rate for $\theta_{i m p}=186.7^{\circ}$. Profile corresponds to the Frame 21 condition in the previous figure.

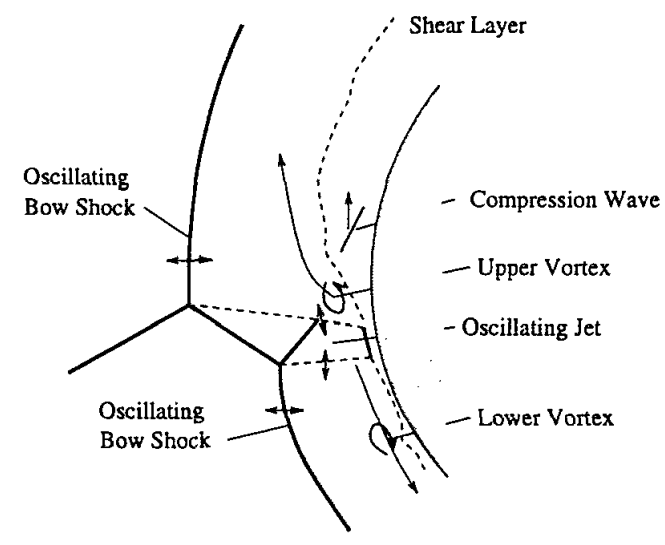

Figure 33: Schematic of the vortex shedding mechanism responsible for the unsteadiness of the flow. The vortices are shed out of phase with respect to each other. The arrows indicate the motions of the flow structures. 
Copyright (C)1996, American Institute of Aeronautics and Astronautics, Inc.

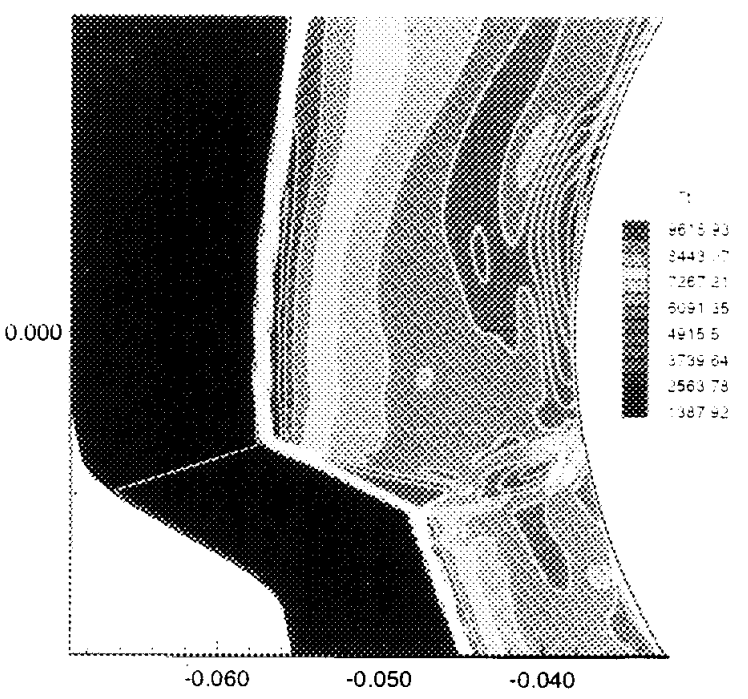

Figure 34: Instantaneous translational temperature iontours.

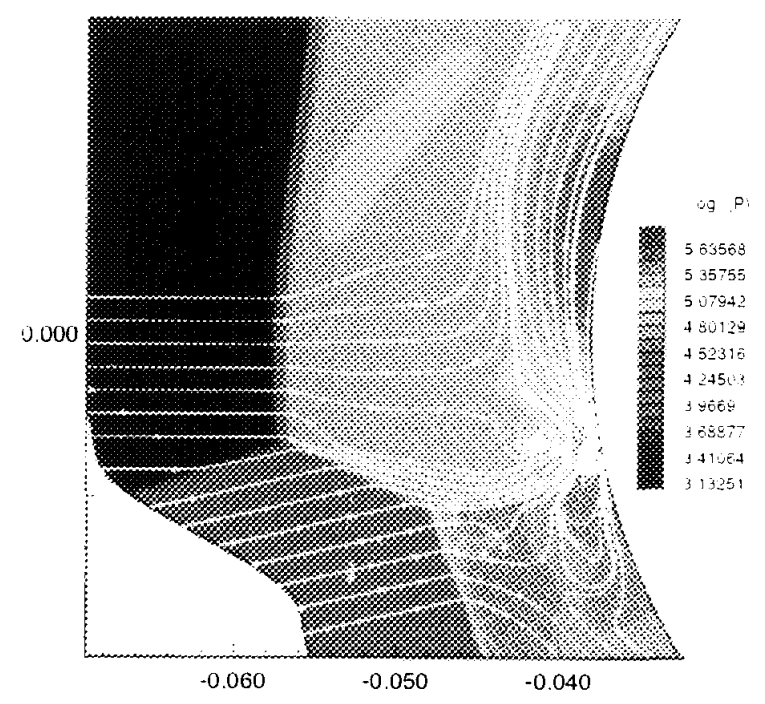

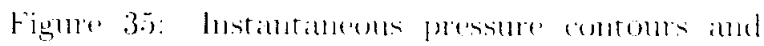
irreimliness.

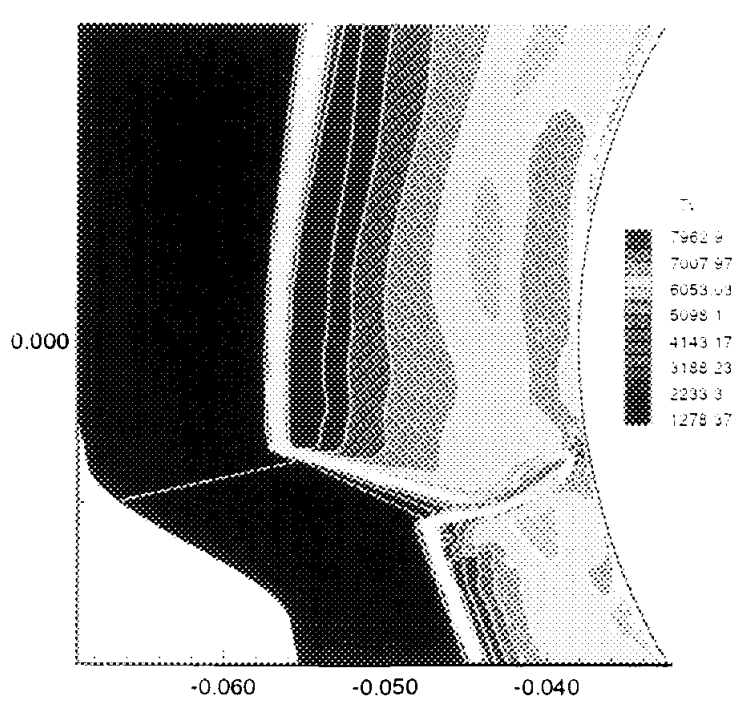

Figure 36: Instantaneons vibrational temperature contouss.

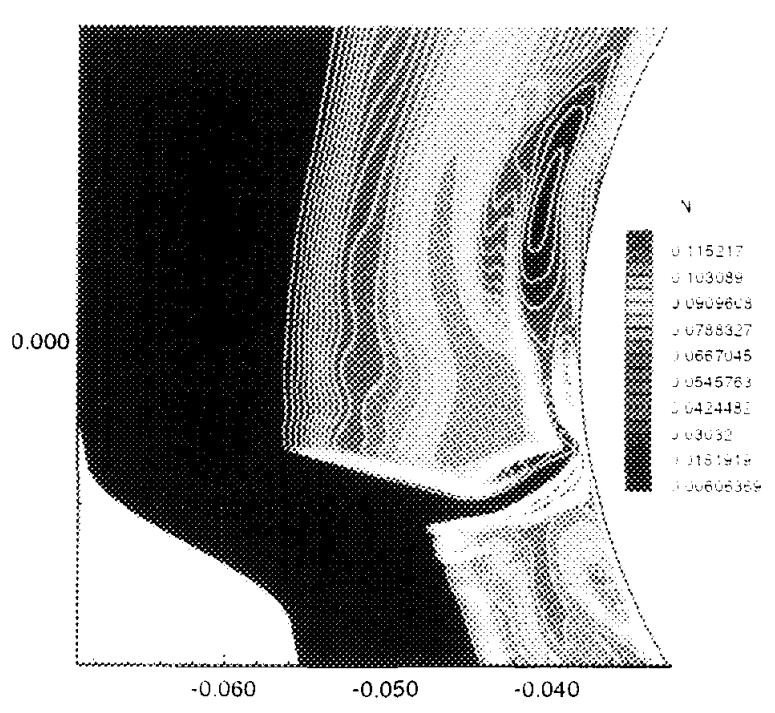

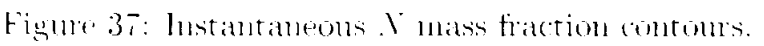




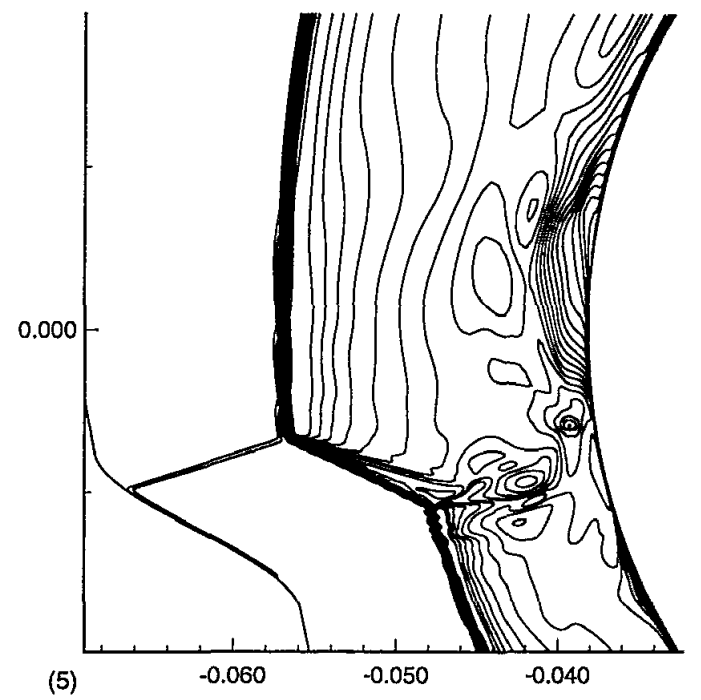

Figure 38: Translational temperature contours: Frame 5

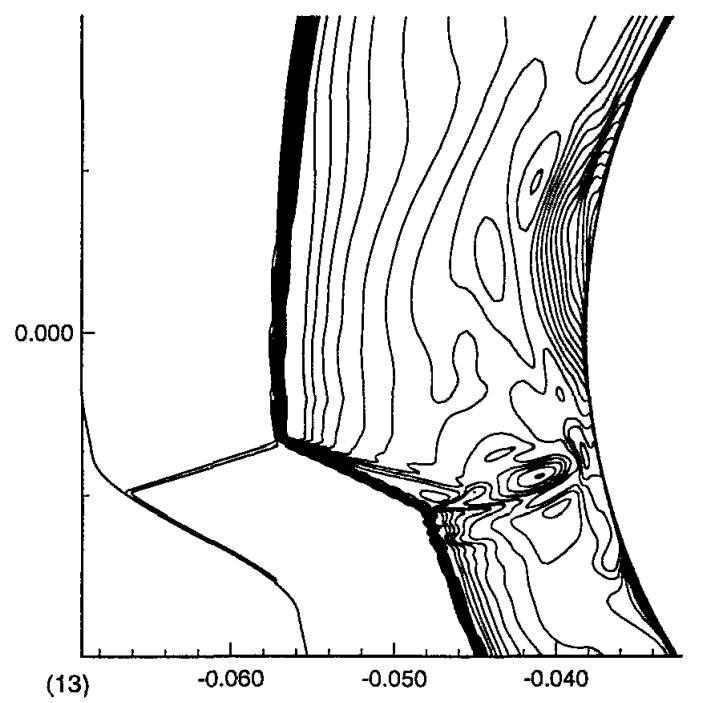

Figure 39: Translational temperature contours: Frame 13

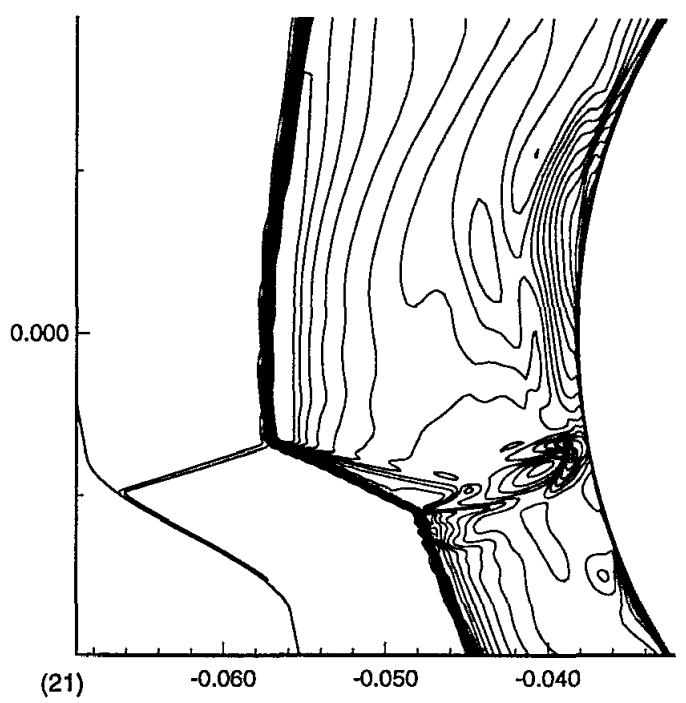

Figure 40: Translational temperature contours: Frame 21

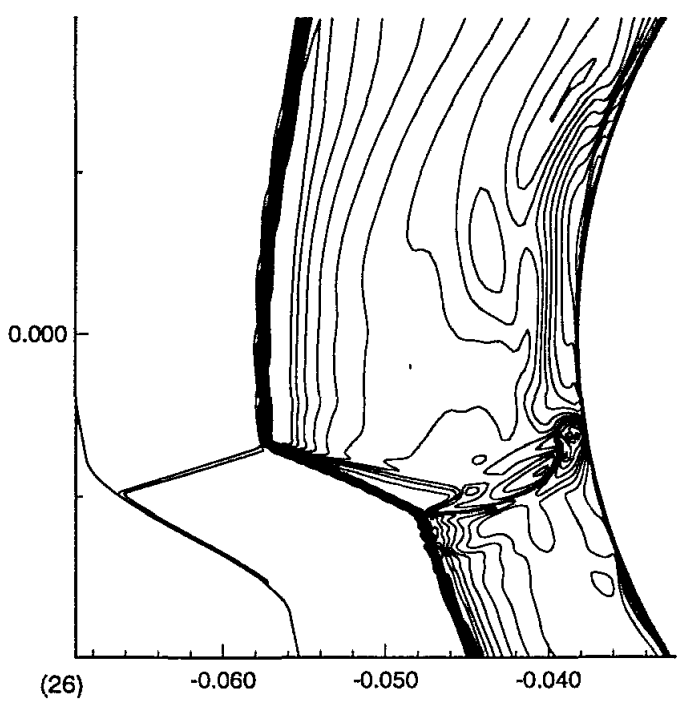

Figure 41: Translational temperature contours: Frame 26 


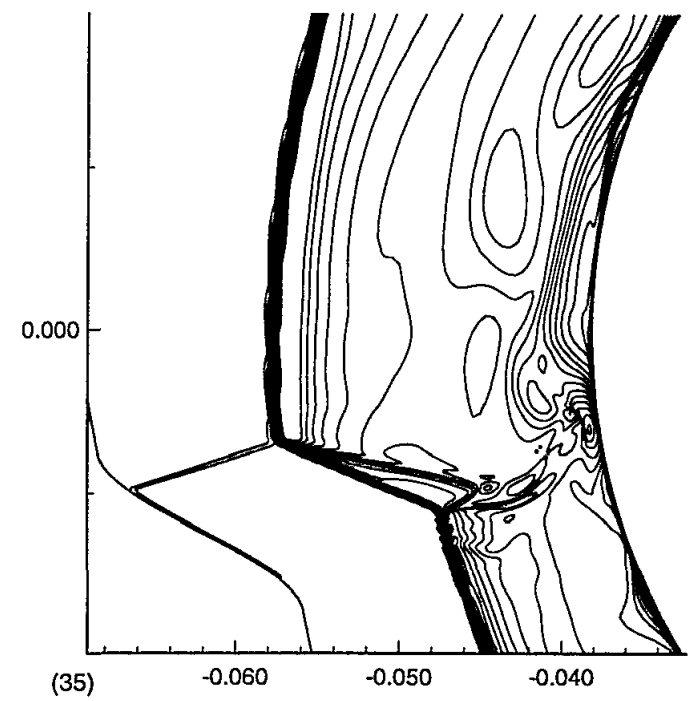

Figure 42: Translational temperature contours: Frame 35

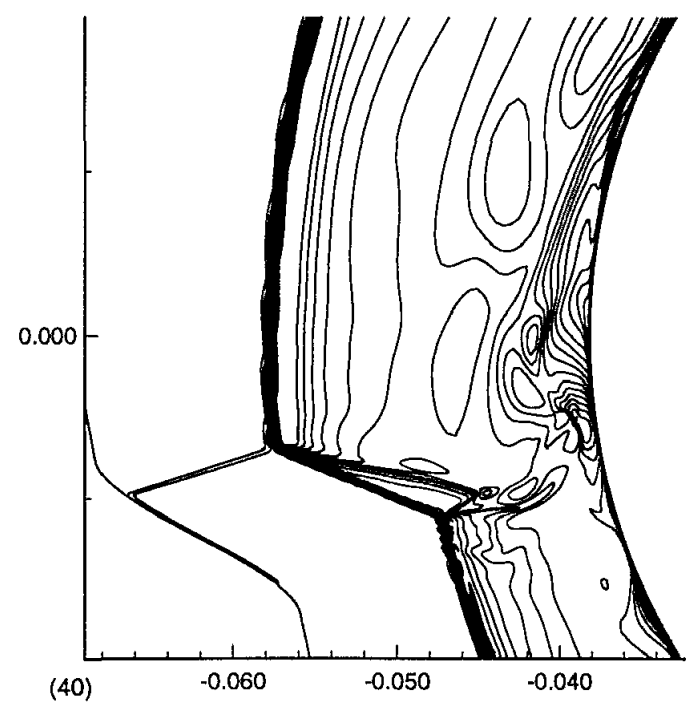

Figure 43: Translational temperature contours: Frame 40

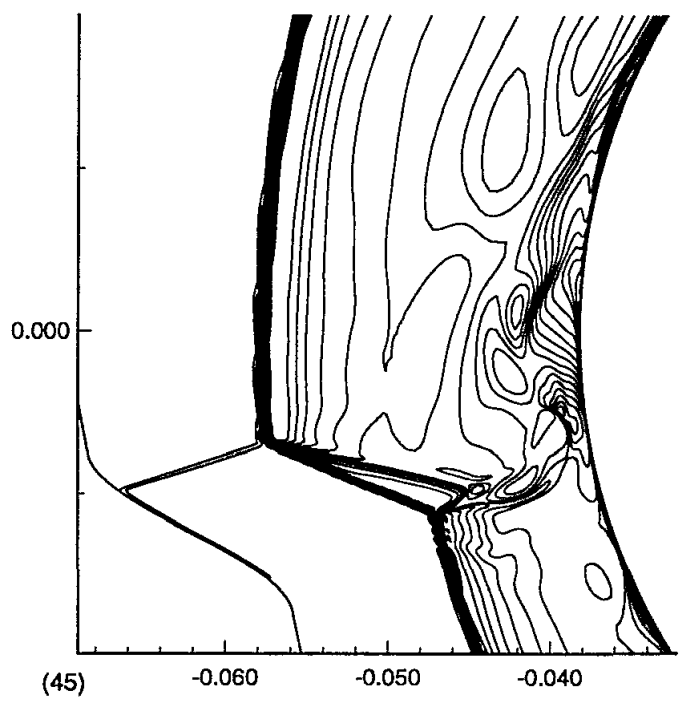

Figure 44: Translational temperature contours: Frame 45

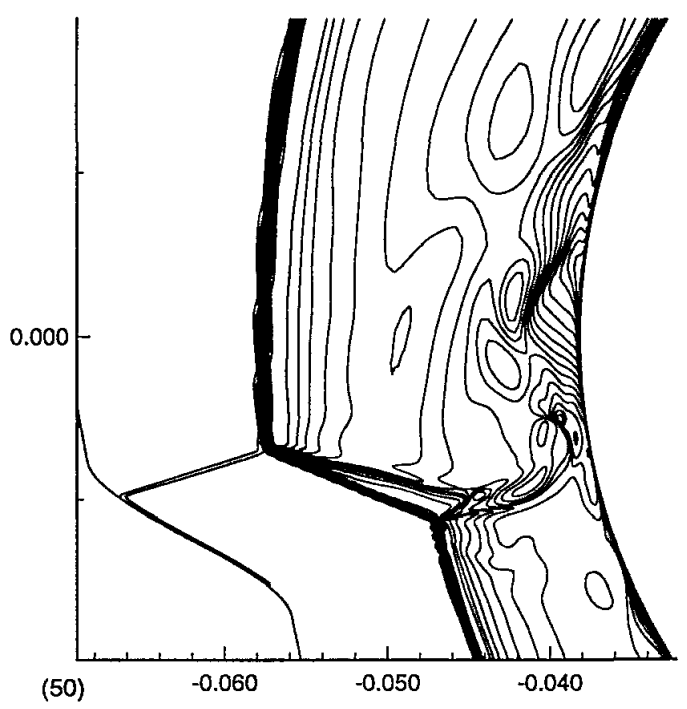

Figure 45: Translational temperature contours: Frame 50 


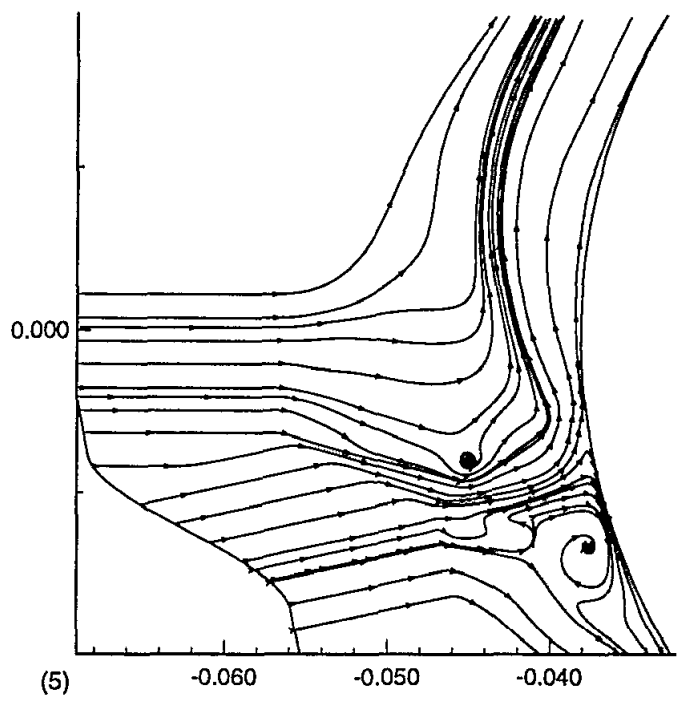

Figure 46: Streamlines: Frame 5

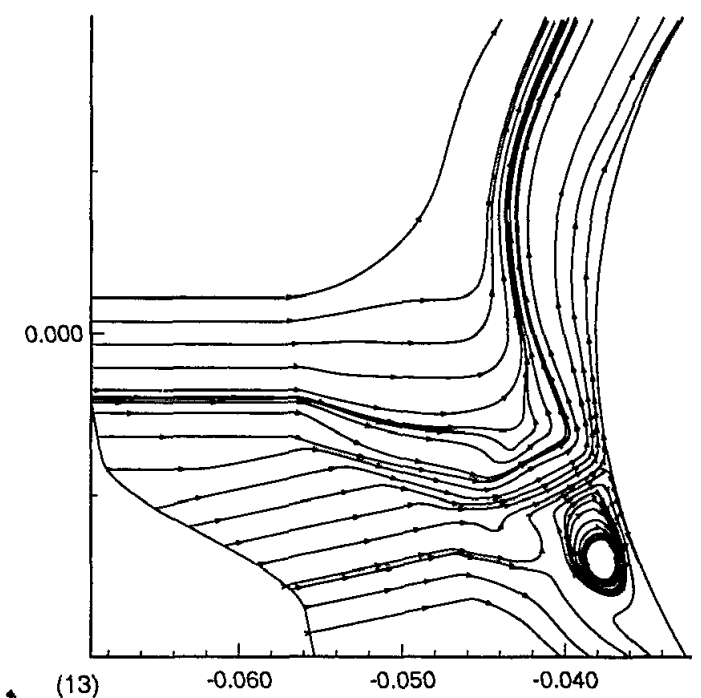

Figure 47: Streamlines: Frame 13

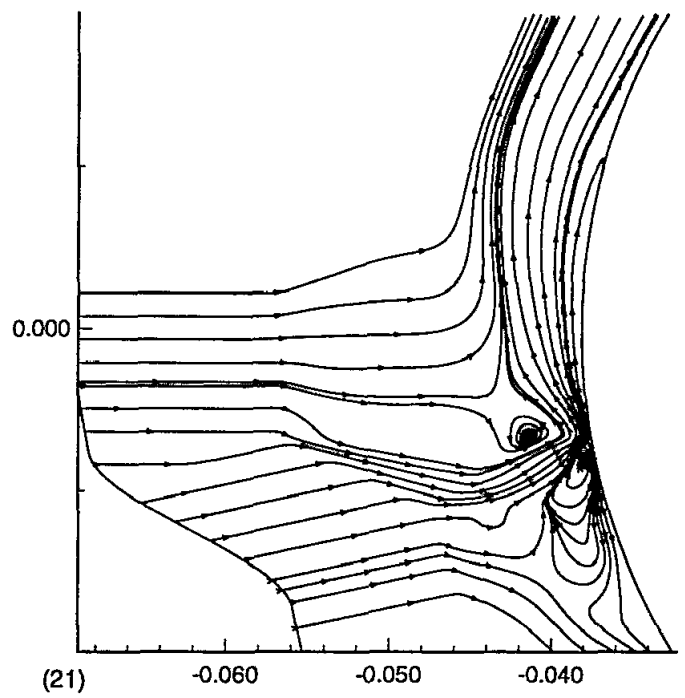

Figure 48: Streamlines: Frame 21

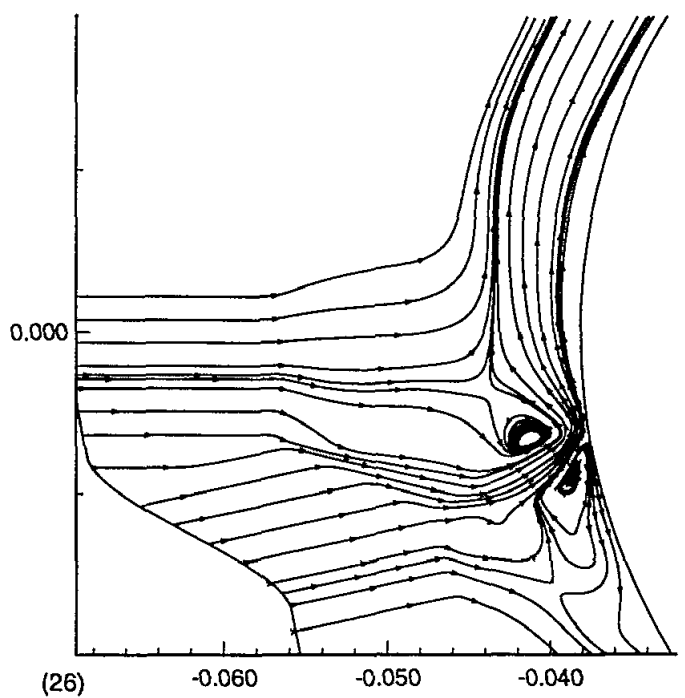

Figure 49: Streamlines: Frame 26 


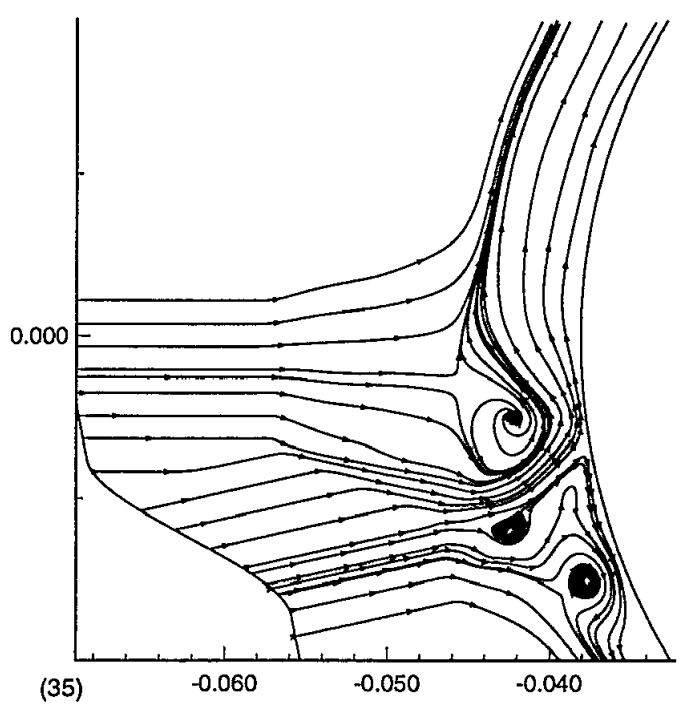

Figure 50: Streamlines: Frame 35

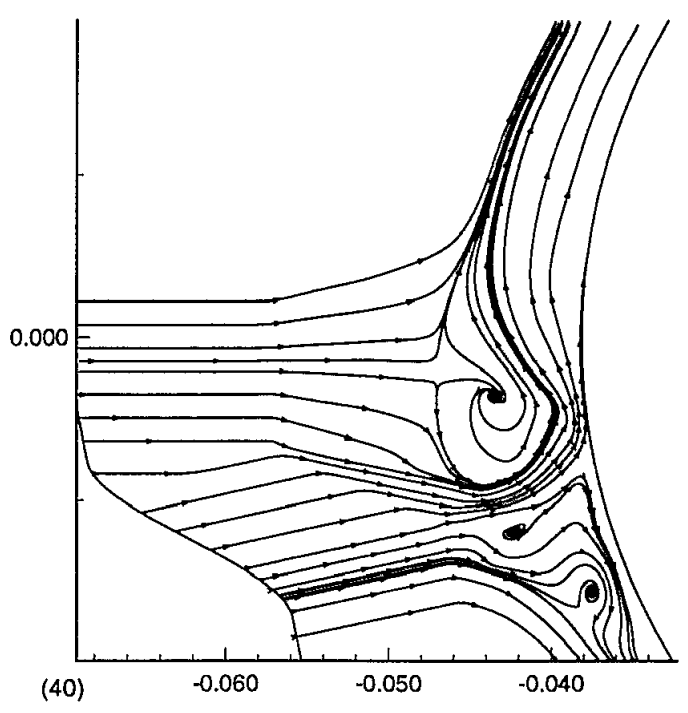

Figure 51: Streamlines: Frame 40

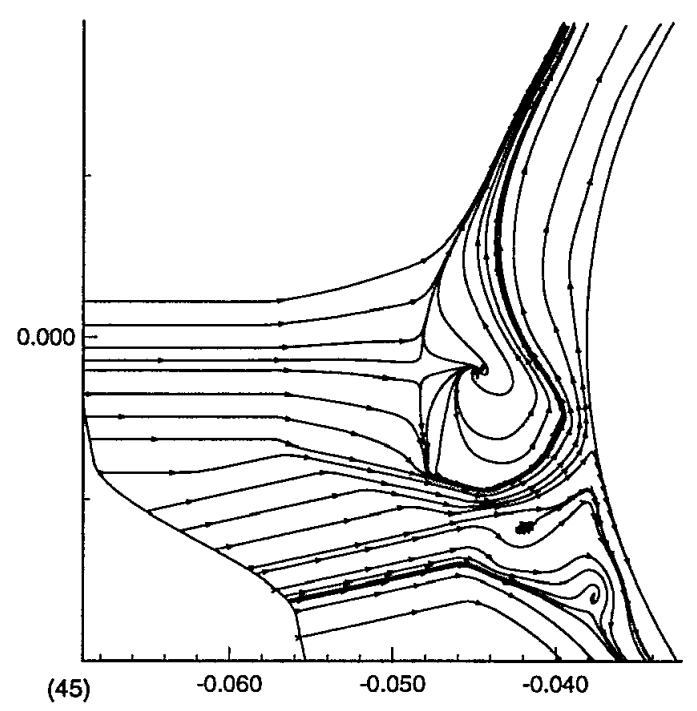

Figure 52: Streamlines: Frame 45

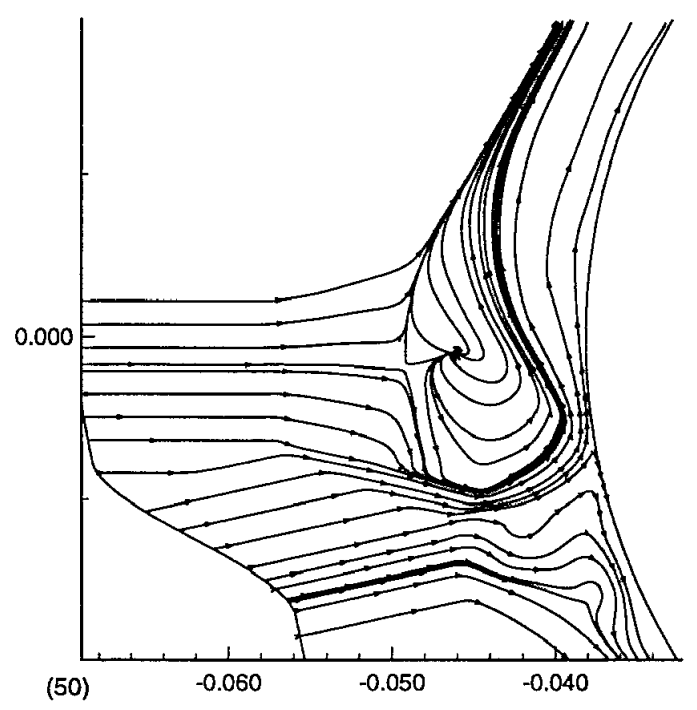

Figure 53: Streamlines: Frame 50 
Copyright (C)1996, American Institute of Aeronautics and Astronautics, Inc.
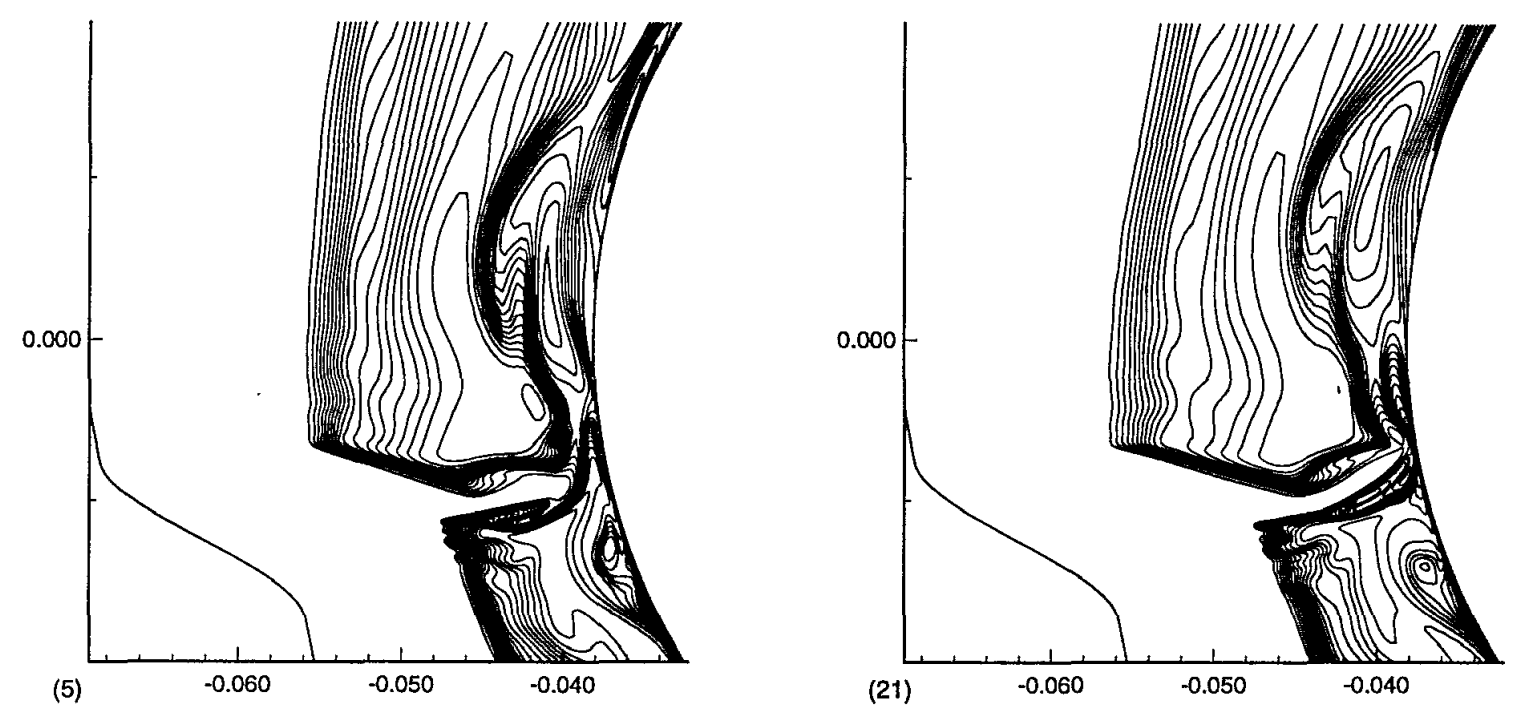

Figure 54: $N$ mass fractions: Frame 5

Figure 56: $N$ mass fractions: Frame 21
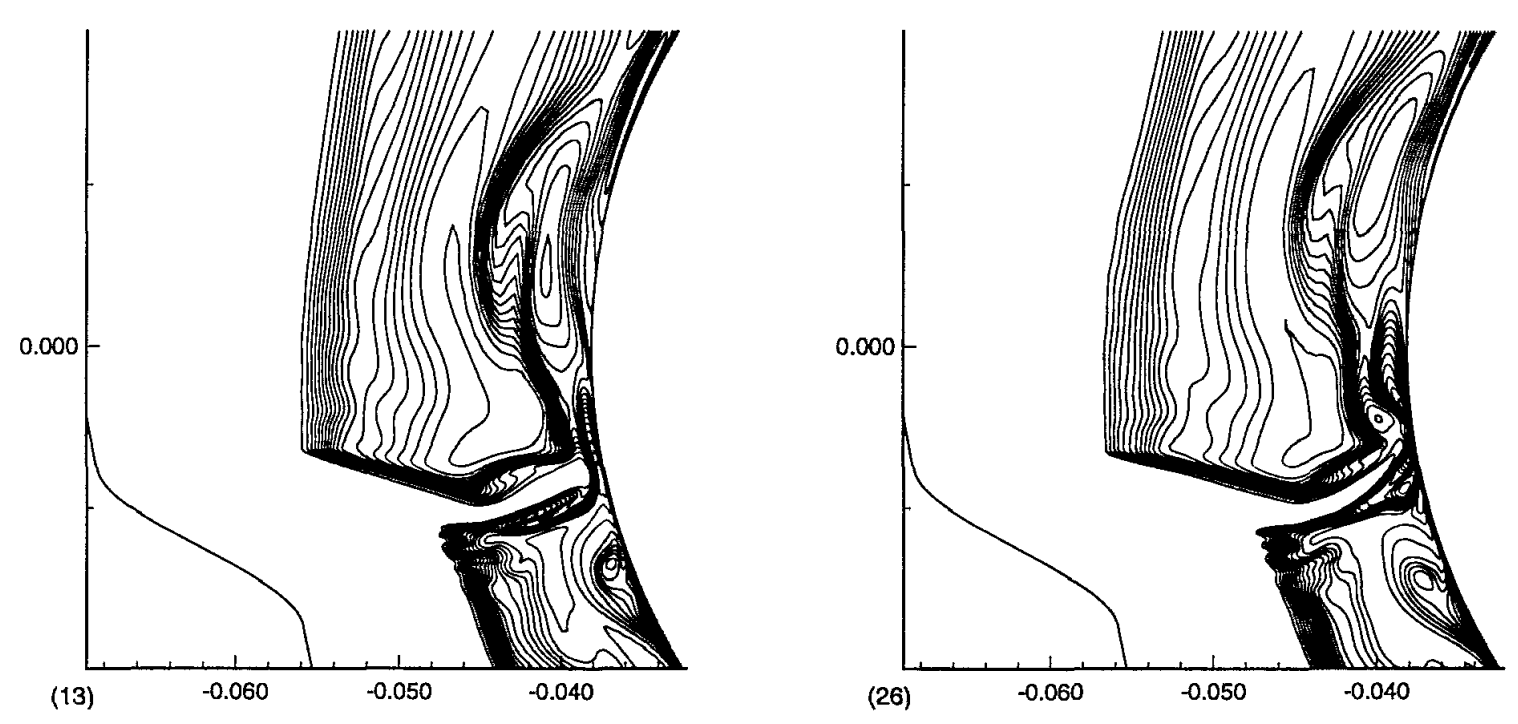

Figure 55: $N$ mass fractions: Frame 13

Figure 57: $N$ mass fractions: Frame 26 
Copyright (C1996, American Institute of Aeronautics and Astronautics, Inc.
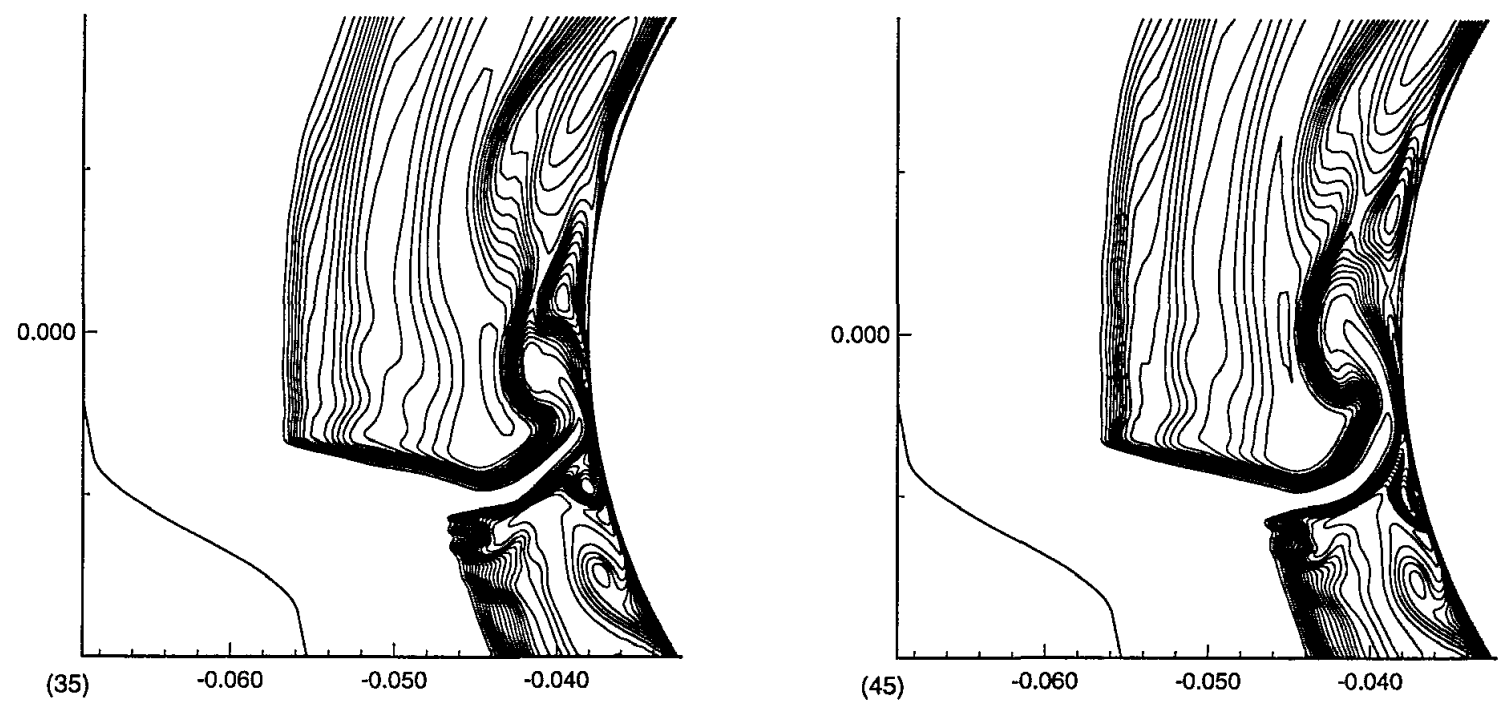

Figure 58: $N$ mass fractions: Frame 35

Figure 60: $N$ mass fractions: Frame 45
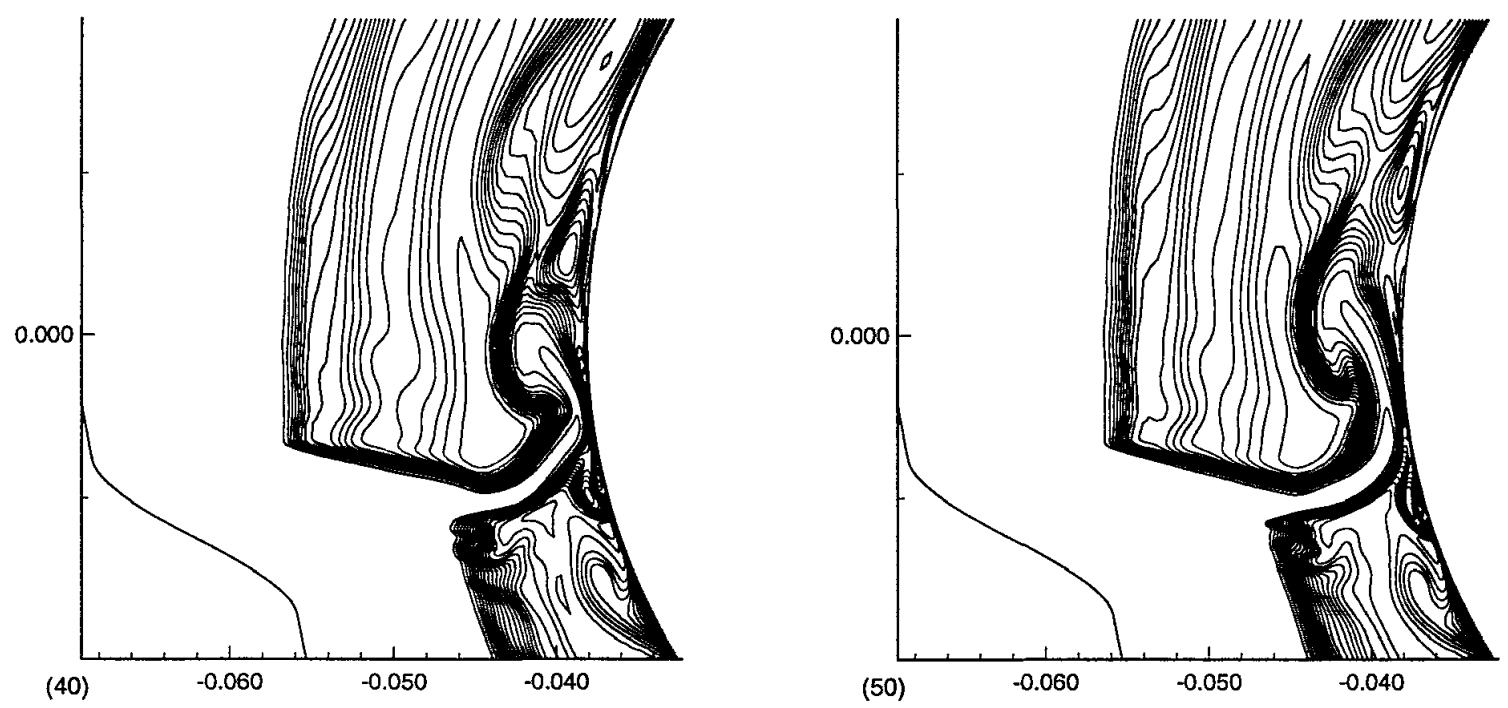

Figure 59: $N$ mass fractions: Frame 40

Figure 61: $N$ mass fractions: Frame 50 


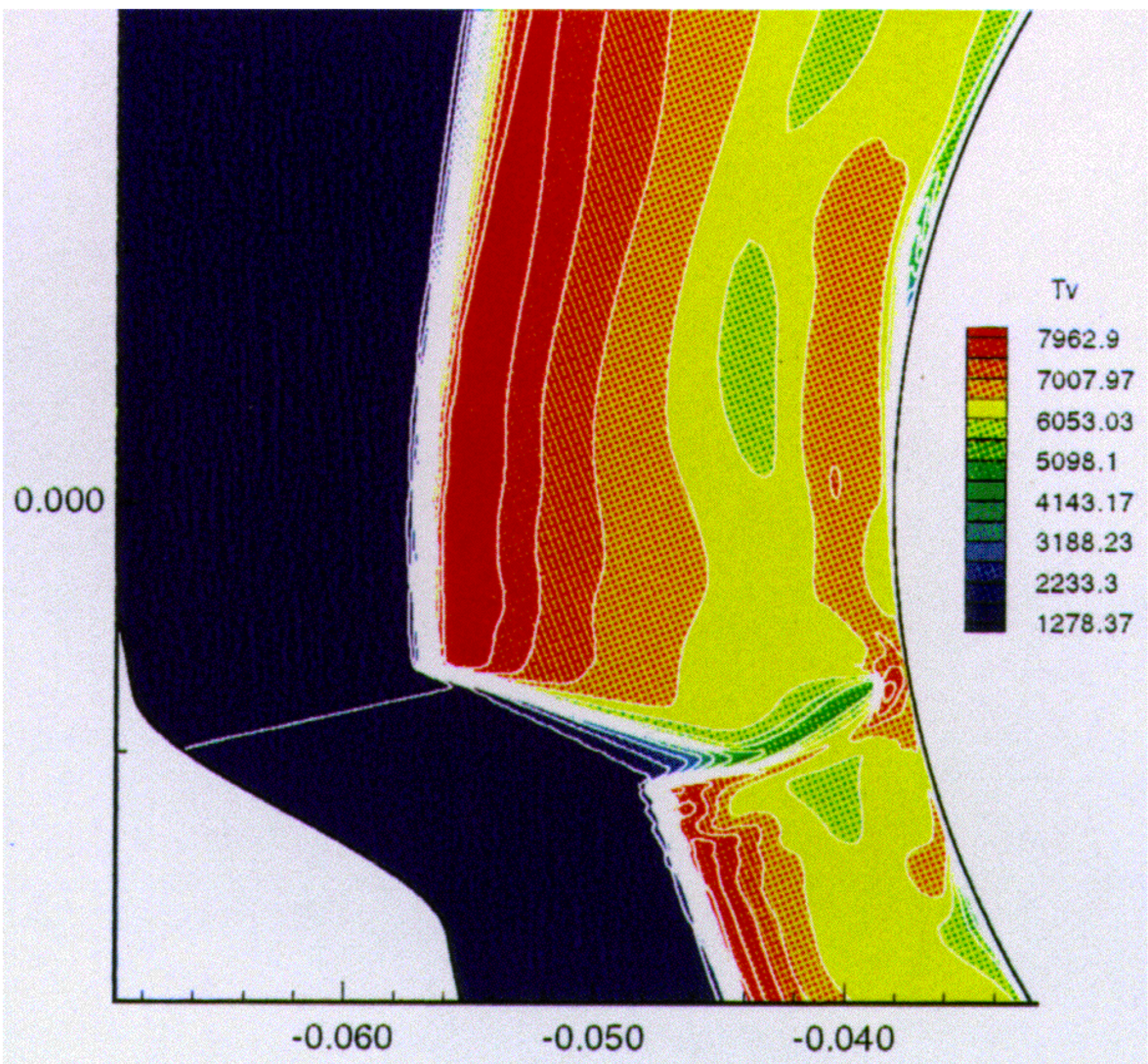




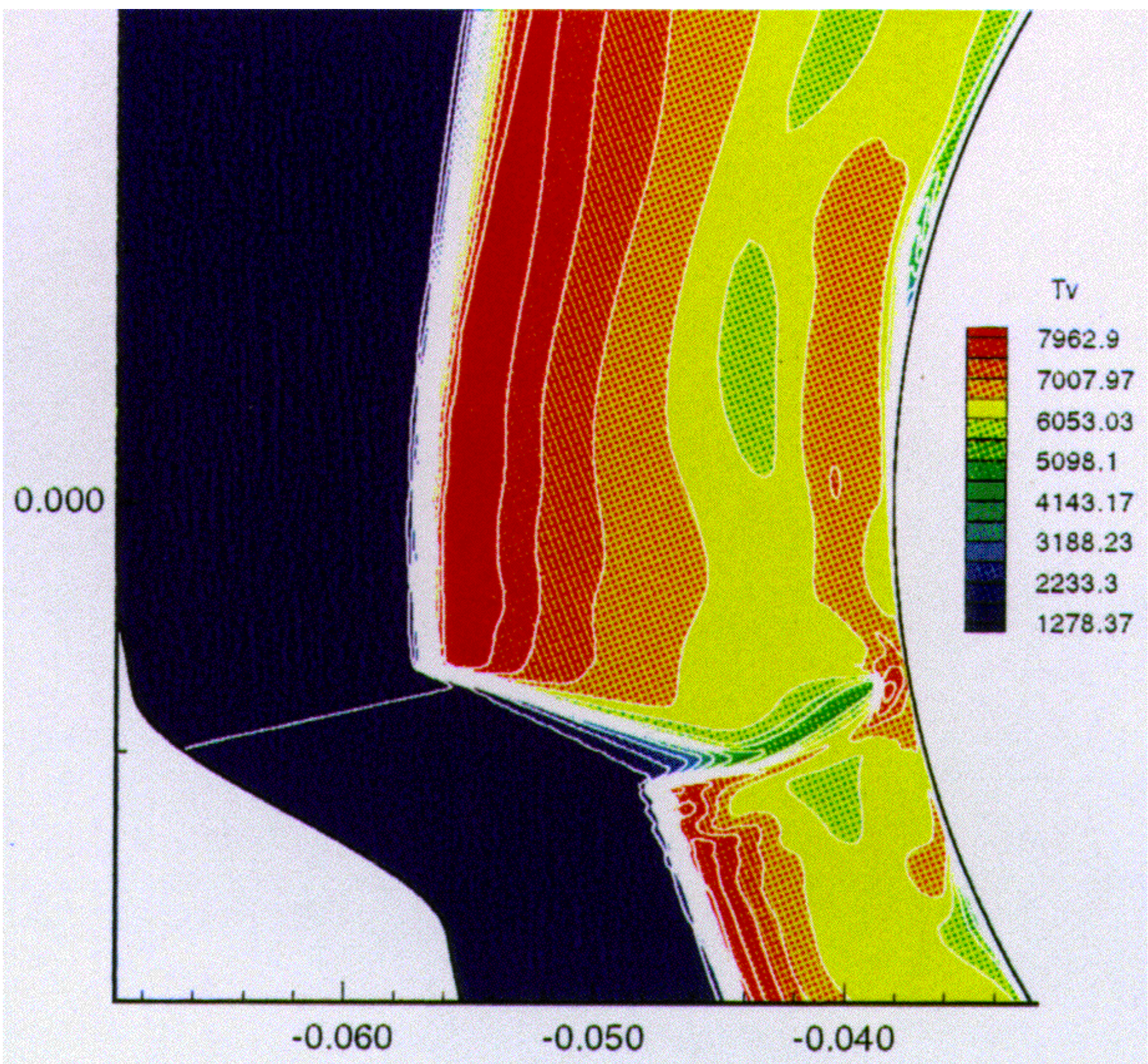




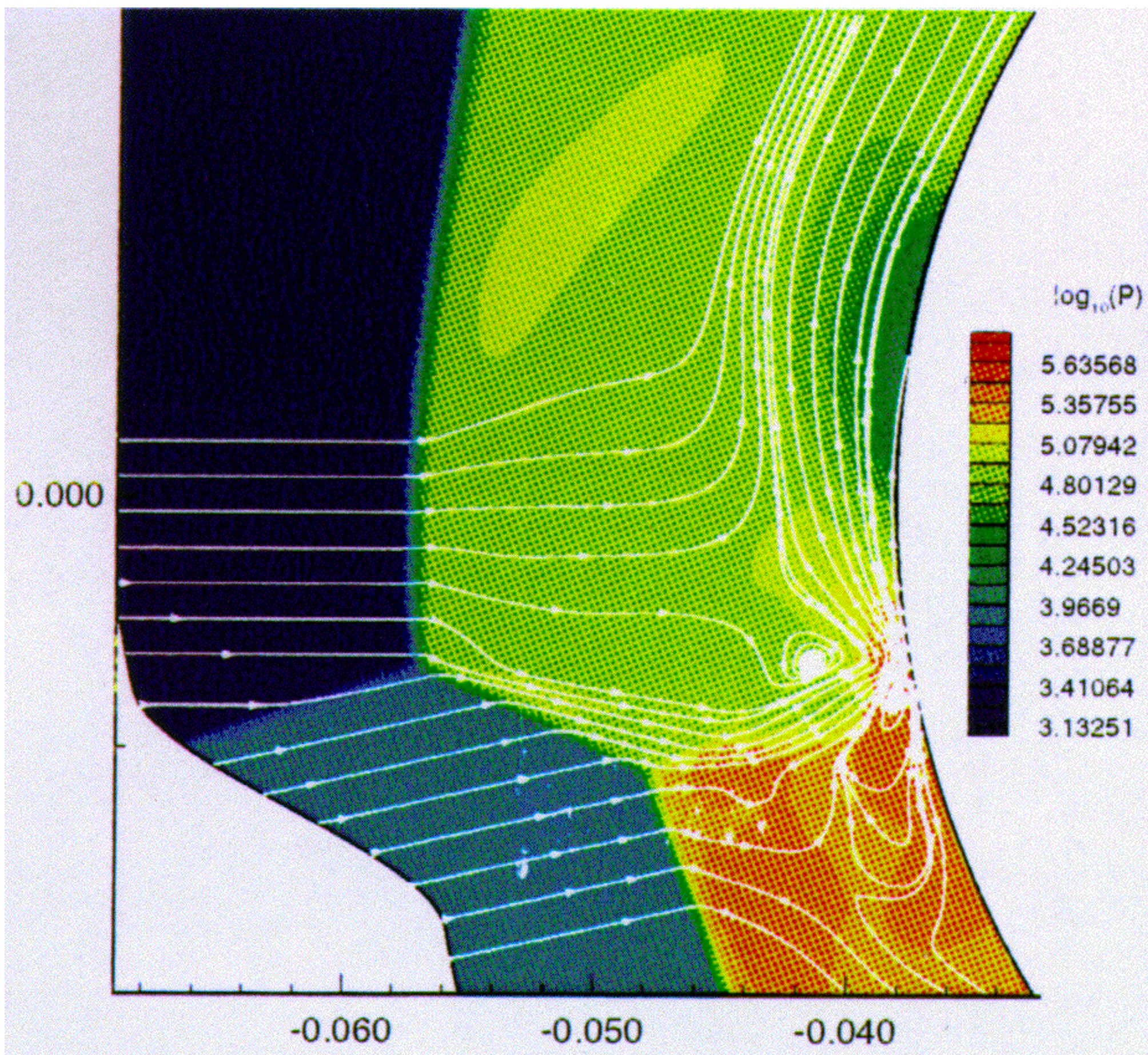




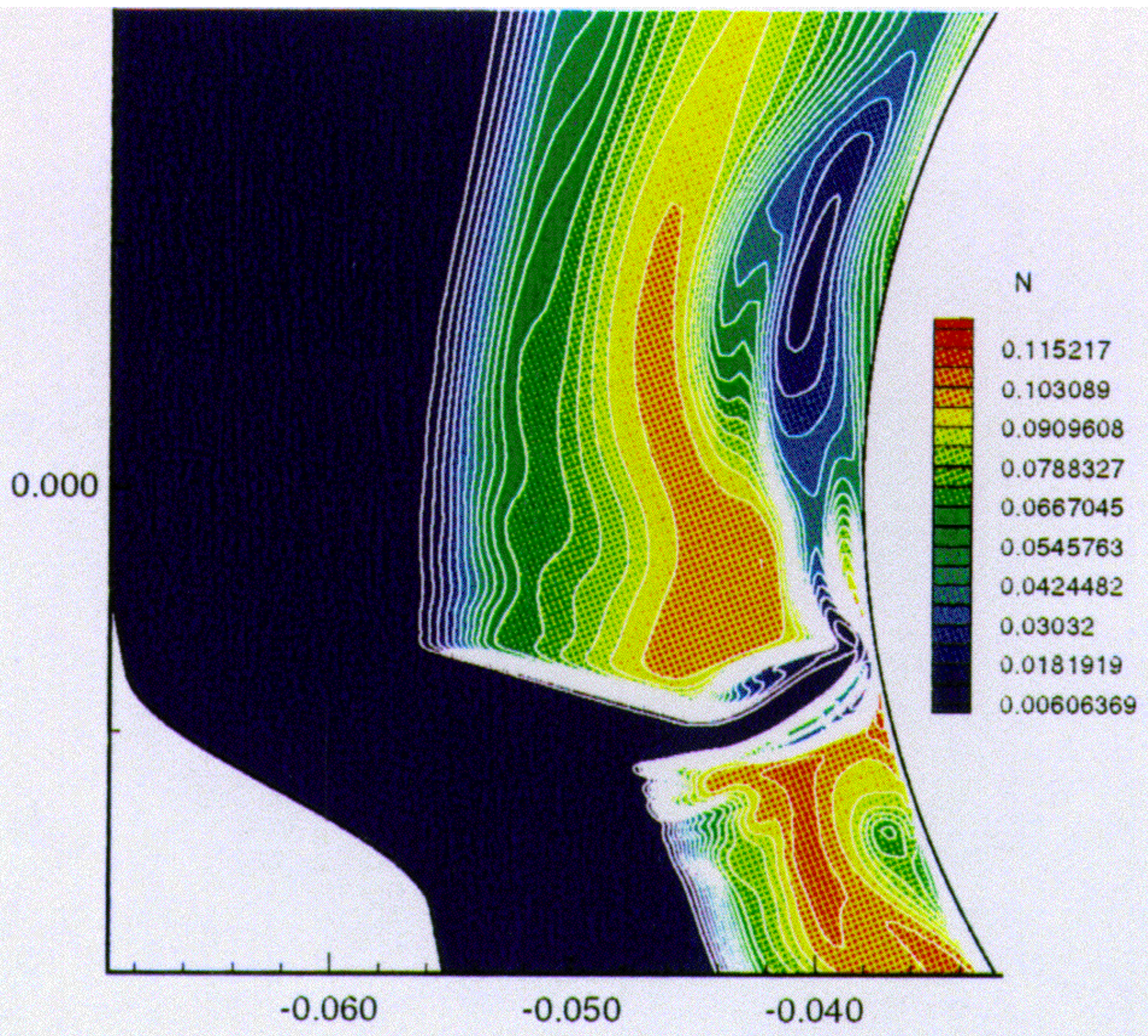

